\title{
Materiali per una raccolta degli scoli greci agli Analitici posteriori di Aristotele
}

\author{
Vito Lorusso \\ I.I.S.S. "F. De Sanctis" - Sant'Angelo dei Lombardi \\ vitolorusso@iissdesanctis.it \\ vito.lorusso@uni-hamburg.de
}

\begin{abstract}
This article aims at mapping the scholia on the first lines from Aristotle's Posterior Analytics A 1. It offers the first edition of the scholia on 71a1-21 from Vaticanus Gr. 241 (13th century), Laurentianus 72,3 (second half of the 13th century) and Laurentianus

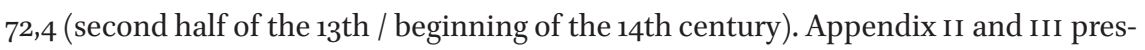
ent the content of a brief writing of Psellus about the Aristotelian Organon and the Praefatio to the Latin translation of Themistius' Paraphrasis to Posterior Analytics written by Hermolaus Barbarus in the $15^{\text {th century. }}$
\end{abstract}

\section{Keywords}

Scholia in Aristotle's Posterior Analytics - Greek manuscripts - Psellus - Hermolaus Barbarus

Nel proemio della Parafrasi degli Analitici posteriori di Aristotele, il retore Temistio (ca. $317-388$ d. C.), amante e studioso della filosofia, ${ }^{1}$ paragona l'opera di chi, nonostante la quantità e qualità dei commenti precedenti, voglia lo stesso produrre un ulteriore lavoro esegetico solo per il gusto di minime varianti nell'interpretazione al tentativo di rifare la celeberrima statua crisoelefantina

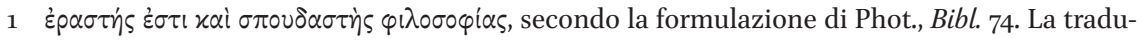
zione italiana citata è tratta da Bianchi-Schiano 2016, 99. 
di Atena Пар日évos scolpita da Fidia nel 438 a.C., soltanto perché pensa di riuscire a rendere meglio un particolare della calzatura:

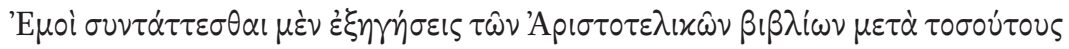

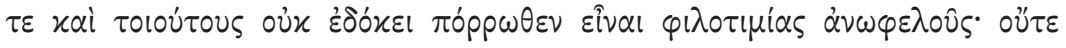

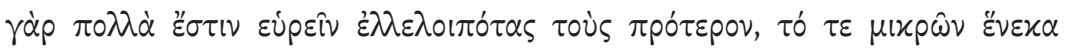

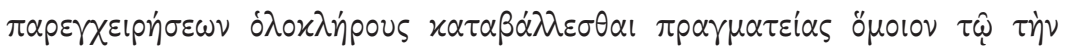

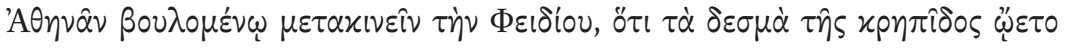

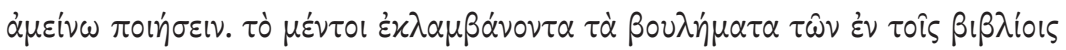

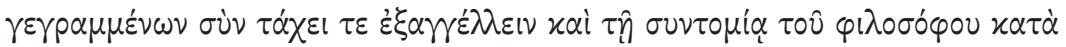

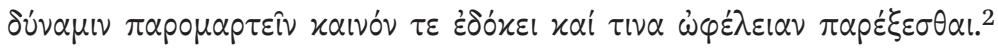

'A me allestire ${ }^{3}$ interpretazioni dei libri di Aristotele dopo tanti e tali (commentatori) non pareva lungi da inutile ambizione. Infatti, non è dato trovare (commentatori) precedenti che abbiano omesso molte spiegazioni, e il mettere per iscritto ${ }^{4}$ intere trattazioni per il gusto di minime varianti (nell'interpretazione) è come voler rifare l'Atena di Fidia perché si pensa di render meglio i legacci del sandalo. ${ }^{5} \mathrm{E}$ però, all'atto di assumermi l'impegno e di svelar presto le intenzioni di ciò che è scritto nei libri (di Aristotele) e di accompagnare (cioè fare un commento perpetuo) per quanto possibile la concisione del Filosofo, (ciò mi) pareva qualcosa di nuovo e latore d'una qualche utilità'.

Purtroppo per noi, salvo frustoli del Commento agli Analitici posteriori di Alessandro di Afrodisia (II-III secolo d.C.) sparsi nei marginalia di codici medievali, nient'altro ci è giunto in tradizione diretta dell'esegesi antica agli Analitici posteriori di Aristotele e dunque l'utilità ( $\grave{\omega} \varphi \hat{c} \lambda \varepsilon i \alpha)$ cui allude Temistio

2 Themist. in APo, CAG V 1, p. 1, 2-10.

$3 \sigma \cup \nu \tau \dot{\alpha} \tau \tau \varepsilon \sigma \theta \alpha \mathrm{l}$ ('ordinare, allestire'): il verbo ricorre anche nel Fedro di Platone, nel passo in cui Socrate e Fedro sottopongono a esame critico il discorso di Lisia sull'amore, cfr. 263 $\mathrm{d}_{7}-\mathrm{e} 3: . .$.

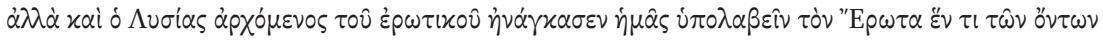

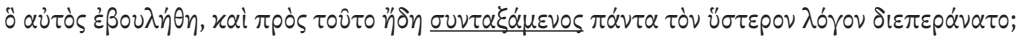

$4 x \alpha \tau \alpha \beta \alpha \lambda \lambda \varepsilon \sigma \theta \alpha$ l ('mettere per iscritto', 'buttar giù il testo', td. 'niederschreiben'): con il medesimo significato, il verbo si incontra molto spesso nei commenti ad Aristotele, cfr. per es.

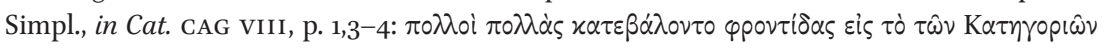

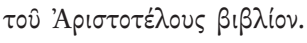

5 Cfr. anche il proverbio latino ne supra crepidam sutor iudicaret, originatosi dalla risposta che, stando a Plinio il Vecchio $(35,36,85)$, il pittore Apelle ebbe a dare a un ciabattino suo critico, vd. anche Tosi 1991, 257-258. 
alla fine della citazione, è oggi meno modesta di quanto allora Temistio stesso volesse far credere. ${ }^{6}$

A voler, infatti, tracciare un quadro della superstite esegesi autoriale agli Analitici posteriori di Aristotele, il risultato è come segue:

- Alessandro di Afrodisia. Del Commento agli Analitici posteriori composto dall' $\dot{\xi} \xi \eta \eta \tau$ '́s per antonomasia rimangono unicamente disiecta membra, tramandati dal Commento di Filopono ${ }^{7}$ oppure sparsi tra gli scoli di alcuni codici greci medievali, per es., il Vaticano gr. 241 siglato con la lettera I fin dall'edizione di Aristotele di Immanuel Bekker. ${ }^{8}$ In questo manoscritto le annotazioni di origine 'alessandrina' sono segnalate dal gen. auctoris 'A $\lambda \varepsilon \xi \dot{\alpha} \nu \delta \rho \circ v$, vd., per es., fol. 135v, 136rv, 137r; ${ }^{9}$

- Temistio, Parafrasi degli Analitici posteriori edita da Max Wallies nel 1900 per i CAG (volumevi); $;^{10}$

- Giovanni Filopono, Commento agli Analitici posteriori edito da Max Wallies nel 1909 per i $C A G$ (volume XIII 3). ${ }^{11}$

L'inscriptio del Commento, ricavata dal Marciano gr. 225, suona nell'edizio-

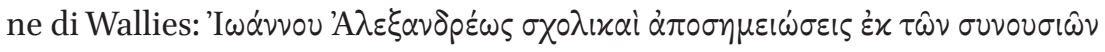

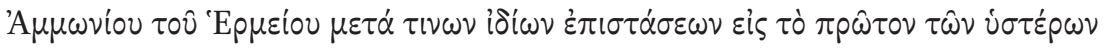

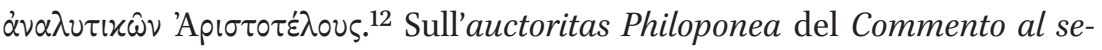
condo libro degli Analitici posteriori sussistono dubbi. ${ }^{13}$ Nel medesimo volume $C A G$ XIII 3, alle pp. 545-603, Wallies editò così anche un commento anonimo al secondo libro degli Analitici posteriori. Molte delle $\dot{\xi} \xi \eta \gamma \eta \dot{\sigma} \sigma ı$

6 Sappiamo per es. che il medico Galeno di Pergamo aveva scritto i $\pi \circ \mu \nu \eta \dot{\eta} \mu \alpha \tau$, non destinati alla pubblicazione, ai due libri degli Analitici posteriori di Aristotele per complessivi undici libri (sei libri per il primo, altri cinque per il secondo). Lo testimonia Galeno stesso nel De libris propriis 14,13 (XIX 42 Kühn = Boudon-Millot 2007, 166,16-19).

7 Cfr. e. g. Phlp. in APo, CAG XIII 3, p. 3,32; 41,2; 62,2.

8 Bekker 1831, IV.

9 Per una raccolta sistematica delle testimonianze e dei frammenti del Commento agli Analitici posteriori di Alessandro di Afrodisia, trasmessi tra gli scholia del Vaticanus I, cfr. Moraux 1979, 61-77.

10 Della Parafrasi manca ancora la traduzione inglese nella serie del King's College di Londra diretta da Richard Sorabji, cfr. www.ancientcommentators.org.uk.

11 Con la pubblicazione di quel volume totum corpus commentariorum in Aristotelem Graecorum ad finem quem voluit Academia Borussica perductum est, come scrive lo stesso Wallies alla p. IV. Il Commento di Filopono è disponibile in traduzione inglese nella serie di Sorabji.

12 Da notare in questa inscriptio la combinazione di 'tradizione' (le note frutto dell'insegnamento di Ammonio, figlio di Ermia) e 'innovazione' (alcune osservazioni proprie).

13 Cfr. Ebbesen 2012, 363. 
raccolte in quelle pagine iniziano con la congiunzione ö $\tau$, che è il tipico modo da parte dei Bizantini di introdurre un escerto; ${ }^{14}$

- Leone Magentino, commento inedito;

- Giovanni Pediasimo (ca. 1270), scoli; ${ }^{15}$

- Eustrazio di Nicea, Commento al secondo libro degli Analitici posteriori; ${ }^{16}$

- Teodoro Prodromo, Commento al secondo libro degli Analitici posteriori; ${ }^{17}$

- notizie su un Commento perpetuo all'Organon di Georgios Pachymeres (1242-ca. 1310) e su una Parafrasi al libro secondo degli Analitici posteriori di Giovanni Cortasmeno (ca. 1370-1436/37), entrambi inediti, danno rispettivamente Cacouros 1994 e Golitsis 2008.18

\section{$2 \quad$ I testimoni manoscritti}

Avviamo con questo lavoro l'esplorazione sistematica degli scoli e delle note interlineari trasmessi dai seguenti diciassette testimoni manoscritti greci degli Analitici posteriori di Aristotele, di età compresa tra il sec. IX e il primo quarto del sec. XIV:

Ambrosianus L 93 sup. saec. IX

Vaticanus Urbinas Gr. 35 saecc. $I X / X$

Vaticanus Barberinianus Gr. 87 saec. $X$

Laurentianus 72,5 saec. X, p. $m$.

Marcianus Gr. 201 a. 954

Parisinus Coislinianus 330 saec. XI

Laurentianus CS 192 saec. XII p. m. vel exeunt.

Vaticanus Gr. 241 saec. XIII

Vaticanus Gr. 244 saec. XIII

Guelferbytanus 24 Gud. Gr. saec. XIII

Laurentianus 72,3 saec. XIII p. m.

Barocci 177 saec. XIII m.

14 "Moraux in 1979 showed beyond reasonable doubt that this anonymous commentary consists to a high degree, perhaps even exclusively, of excerpts from Alexander of Aphrodisias' lost commentary", cfr. Ebbesen 2012, 363.

15 Editi da De Falco 1926 e 1928.

16 Edizione in CAG XXI.

17 Edito da Cacouros 1992.

18 In generale, per una tipologia dei commenti bizantini ad Aristotele il contributo più sistematico, a mio avviso, è quello di Michele Trizio previsto per http://encyclopedie-humanisme.com. 
Monacensis Gr. 222 saec. XIII exeunt.

Princetonensis MS. 173 saec. XIII exeunt.

Vaticanus Barberinianus Gr. 164 saec. XIII exeunt.

Marcianus Gr. 202 saec. XIII exeunt.

Laurentianus 72,4 saecc. XIII p. m. / XIV ineunt.

Gli scoli di tre di questi manoscritti (Vaticanus Gr. 241, Laurentianus 72,3 e Laurentianus 72,4 ) relativi alla sezione $7101-21$ sono stati trascritti e vengono qui per la prima volta pubblicati con apparato critico in cui sono evidenziate le divergenze rispetto al testo del Commento di Filopono, ove esso è presente.

\subsection{Scholia Graeca in Aristotelis Analytica posteriora A 1 (71a1-21) $e$ codicibus Vaticano Gr. 241, Laurentiano 72,3 et Laurentiano 72,4: editio princeps}

\section{Vaticanus Gr. 241}

f. 113r:

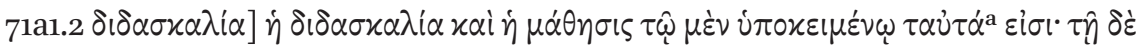

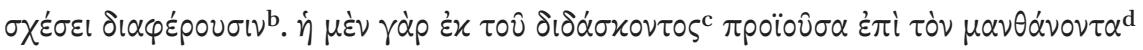

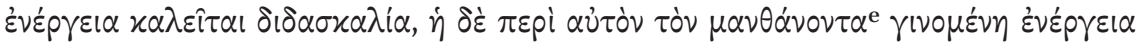

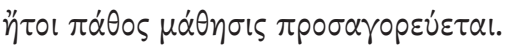

Schol. e Phlp. in APo CAG XIII 3, p. 4,16-17 et 4,20-22 excerpt., pos. in mg. ext. et nigro atram. scr.

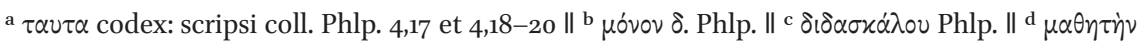

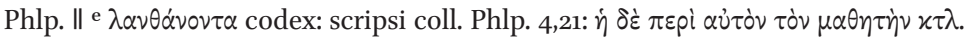

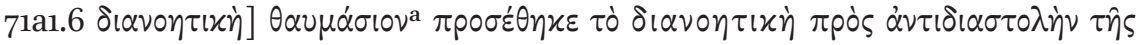

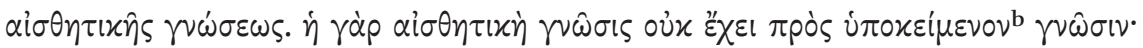

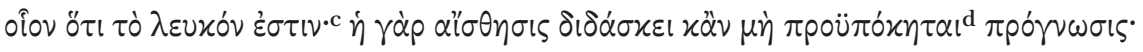

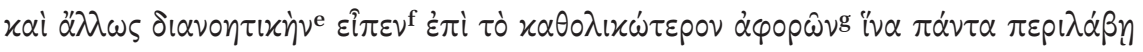
$\sigma 0 \lambda \lambda \circ \gamma 1 \sigma \mu \sigma^{\mathrm{h}}$.

Schol. e Phlp. in APo CAG XIII 3, p. 4,29-5,2 et 5,4-5 excerpt., pos. in mg. int. et nigro atram. scr.

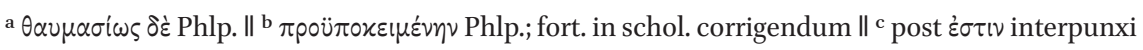

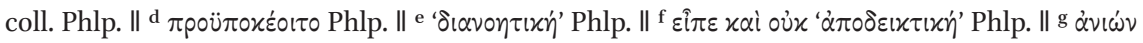

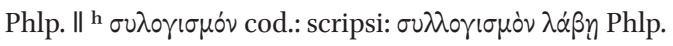

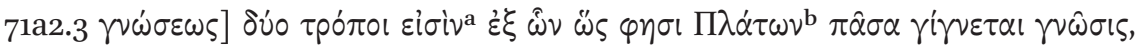

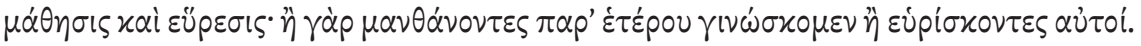

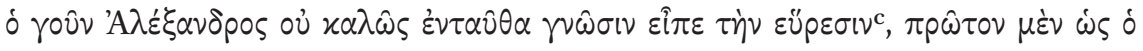




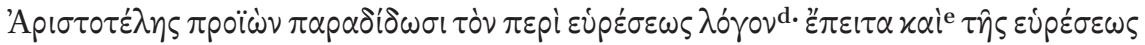

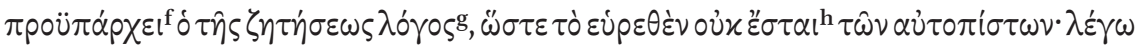

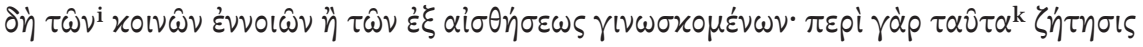

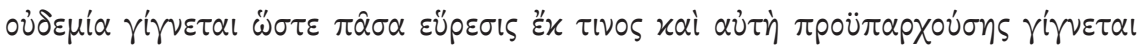

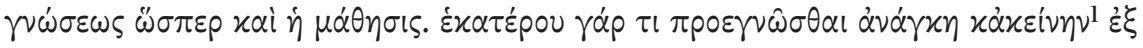

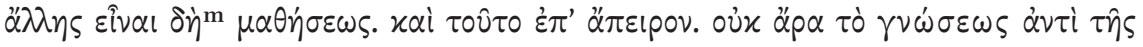

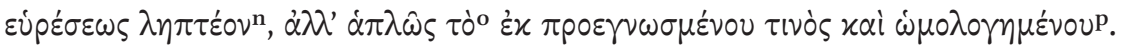

Schol. e Phlp. in APo CAG XIII 3, p. 3,30-4,1; 4,3-6; 4,7-9; 4,10-11 et 4,12-14 excerpt., pos. in mg. ext. iuxta schol. ad 71a1.2 et nigro atram. scr.

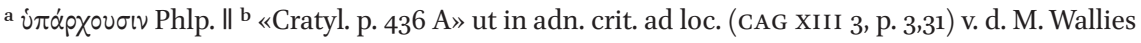

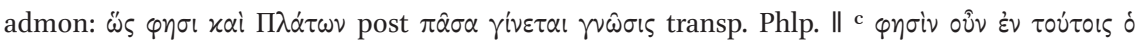

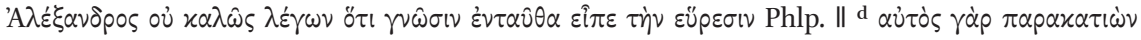

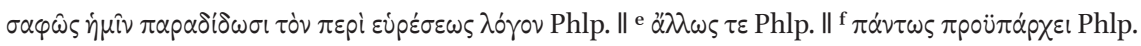

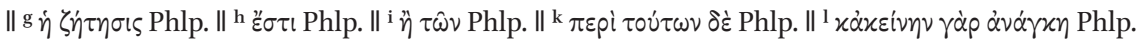

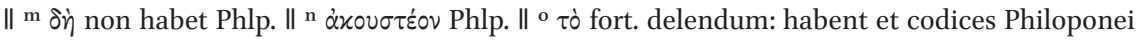
U (Marcianus Gr. 225) et V (Vaticanus Gr. 247) sec. adn. crit. ad loc. (CAG XIII 3, p. 4,14) II p

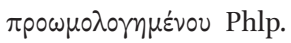

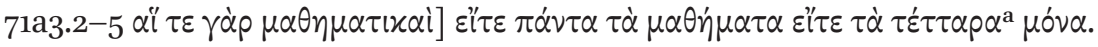

Schol. i. l. pos. et fusco atram. scr.

a $\delta$ codex. De his quattuor disciplinis, i. e. philosophia, astronomia, geometria et arithmetica, vd. e. g. Ammon. in Porph. CAG IV 3, p. 6,25-27; 7,2-4 et 7,13-14.

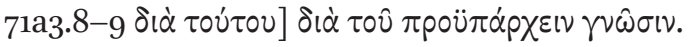

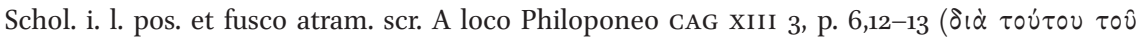

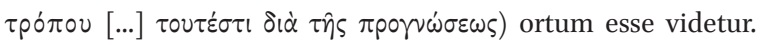

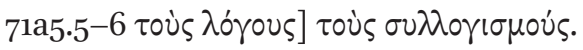

Schol. i. l. pos. et fusco atram. scr.

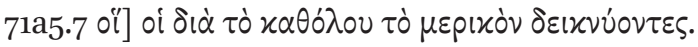

Schol. i. l. pos. et fusco atram. scr.

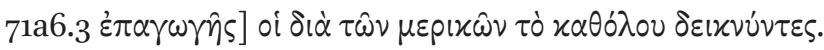


Schol. i. l. pos. et fusco atram. scr.

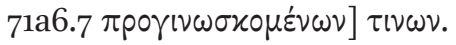

Schol. i. l. pos. et fusco atram. scr.

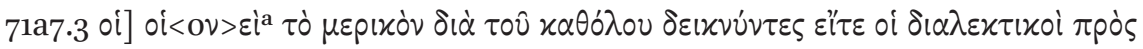
$\delta 1 \alpha \lambda \varepsilon \gamma \circ \mu \varepsilon \dot{v} \omega \omega \nu$.

Schol. i. l. pos. et fusco atram. scr.

a oi हi codex ut vid.: supplevi

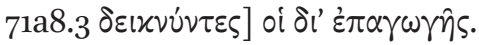

Schol. i. l. pos. et fusco atram. scr.

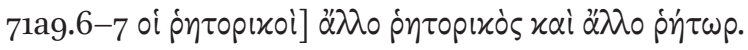

Schol. in mg. int. et fusco atram. scr.

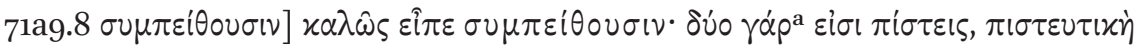

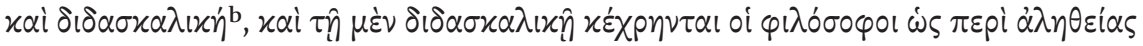

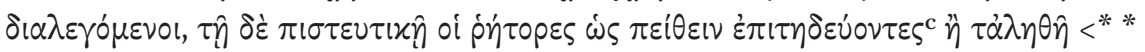
$*$ d.

Schol. e Phlp. in APo CAG XIII 3, p. 7,1-5 loco Platonico in Grg. 452e9-453a7 nitente (vd. adn. crit. ad CAG XIII 3, p. 7,2) excerpt., i. l. pos. et fusco atram. scr.

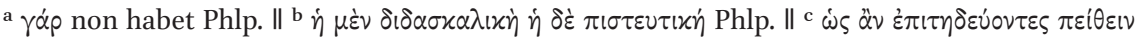
Phlp. II d scriptura fere evanida: $\pi \rho \circ \beta \alpha \dot{\lambda} \varepsilon \varepsilon \sigma \theta \alpha$ Phlp.

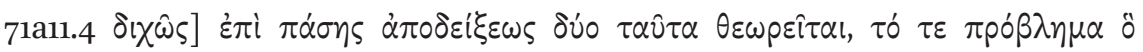

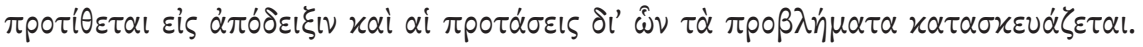

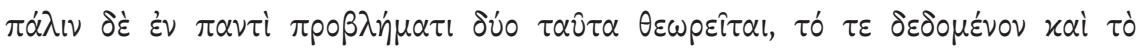

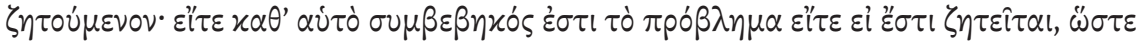

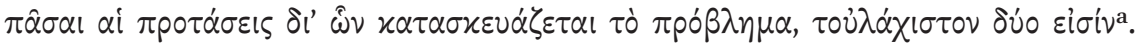

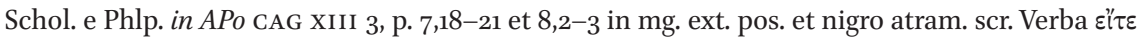

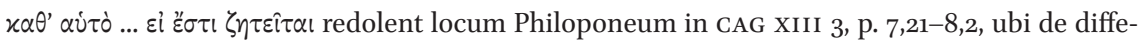

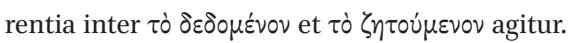

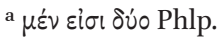




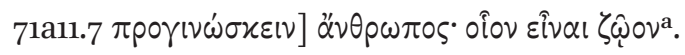

Schol. in mg. int. et fusco atram. scr.

a $\tau \dot{\jmath} \zeta \hat{\omega}$ ov codex ante corr.

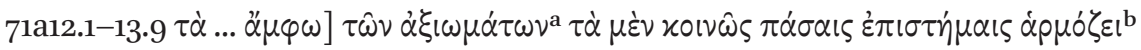

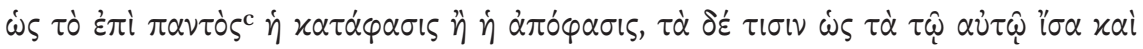

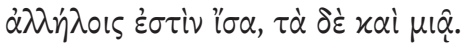

Schol. e Phlp. in APo CAG XIII 3, p. 10,27-32 in mg. int. et nigro atram. scr.

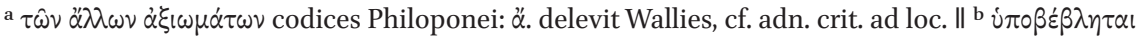

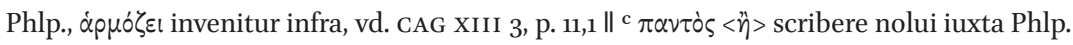

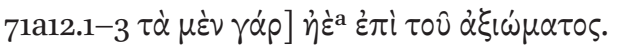

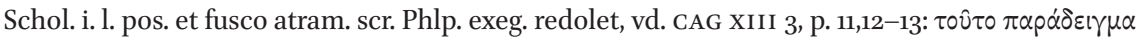

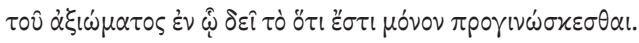

a incert.

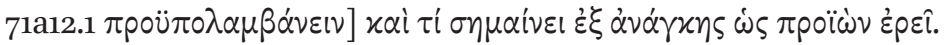

Schol. i. l. pos. et fusco atram. scr.

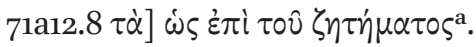

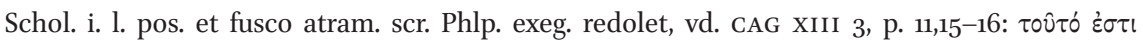

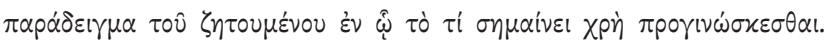

a incert.; litterae $\zeta \eta \tau$ fere evanidae

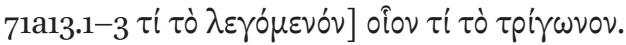

Schol. in mg. int. pos. et fusco atram. scr. Ex Aristotelico loco APo 71a14-15 ortum esse videtur.

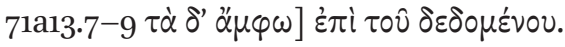

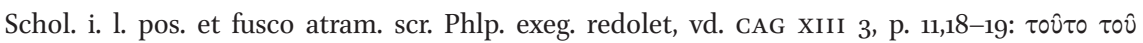

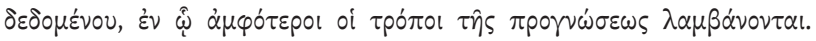

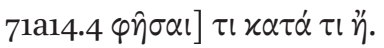


Schol. i. l. pos. et fusco atram. scr.

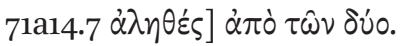

Schol. i. l. pos. et fusco atram. scr.

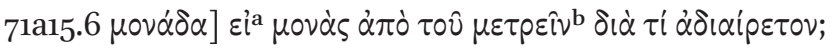

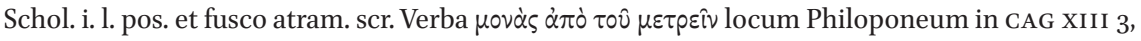

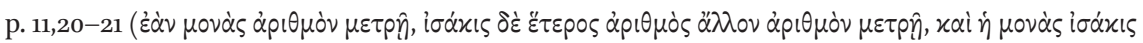

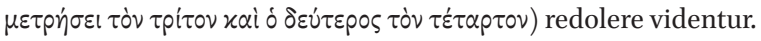

$\mathrm{a}$ $\varepsilon$ i fere evanidum $\| \mathrm{b}$ incert.

\section{Laurentianus 72,3}

f. 69v:

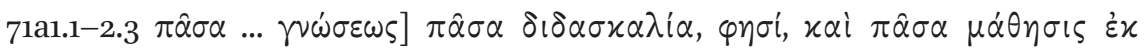

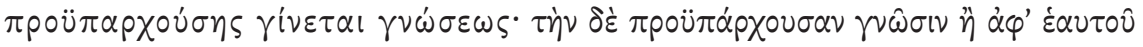

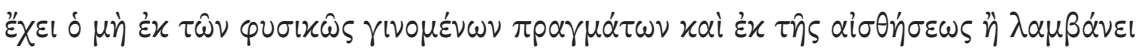

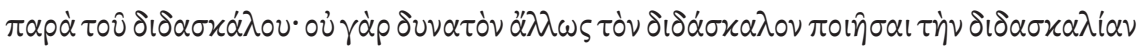

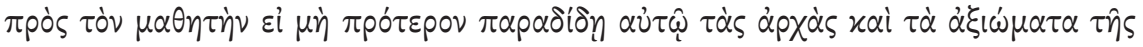

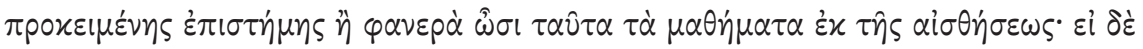

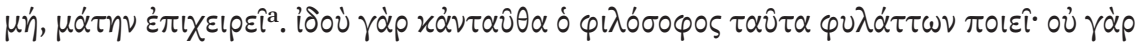

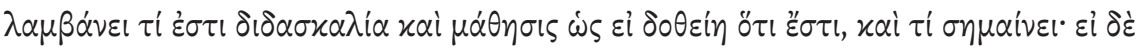

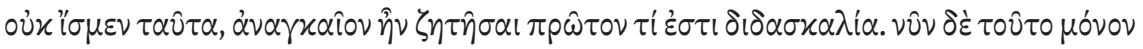

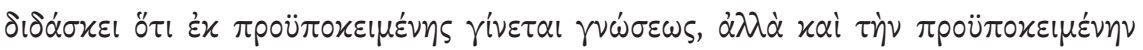

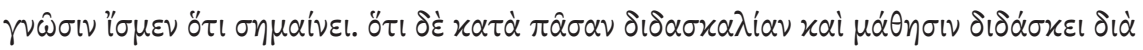

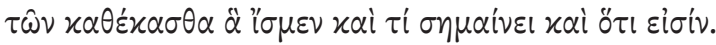

Schol. in mg. ext. pos. et nigro atram. scr.

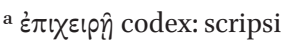

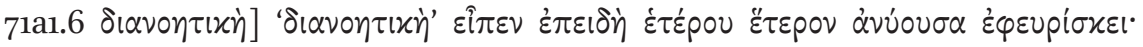

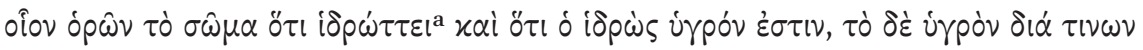

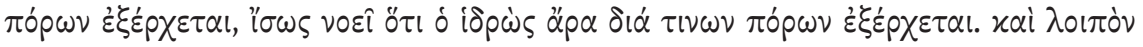

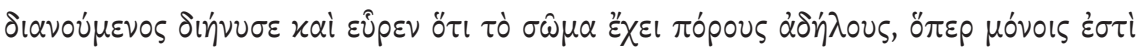

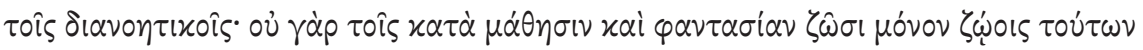

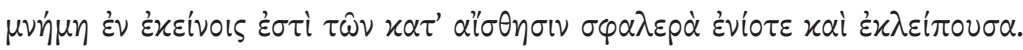

Schol. in mg. ext. pos. et nigro atram. scr.

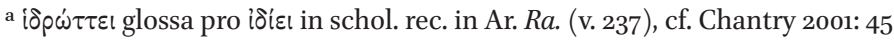




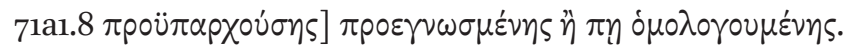

Schol. i. l. pos. et nigro atram. scr. E loco Philoponeo CAG XIII 3, p. 4,14 ortum esse videtur.

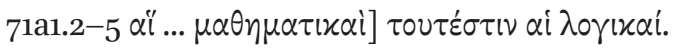

Schol. e Phlp. in APo CAG XIII 3, p. 6,12 excerpt., i. l. pos. et nigro atram. scr.

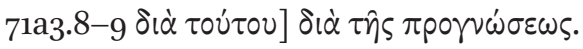

Schol. e Phlp. in APo CAG XIII 3, p. 6,13 excerpt., i. l. pos. et nigro atram. scr.

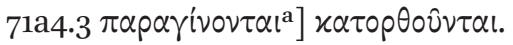

Schol. e Phlp. in APo CAG XIII 3, p. 6,13 excerpt., i. l. pos. et nigro atram. scr.

a yivovtal codex

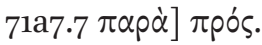

Schol. i. l. et nigro atram. scr.

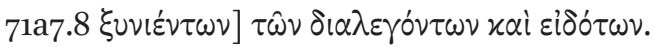

Schol. i. l. pos. et nigro atram. scr.

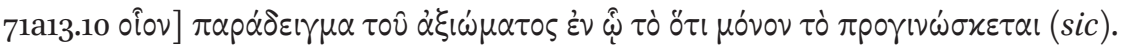

Schol. e Phlp. in APo CAG XIII 3, p. 11,12-13 excerpt., i. l. pos. et nigro atram. scr.

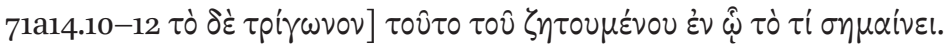

Schol. e Phlp. in APo CAG XIII 3, p. 11,15 excerpt., i. l. pos. et nigro atram. scr.

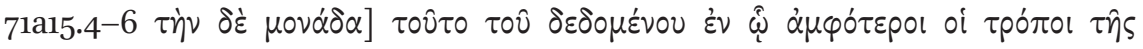
$\pi \rho \circ \gamma \omega \dot{\omega} \sigma \varepsilon \omega \varsigma \lambda \alpha \mu \beta \alpha \dot{\alpha} \sigma \nu \tau \alpha$ l.

Schol. e Phlp. in APo CAG XIII 3, p. 11,18-19 excerpt., i. l. pos. et nigro atram. scr.

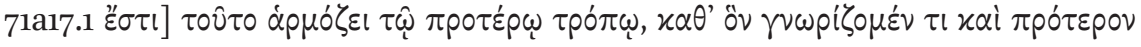

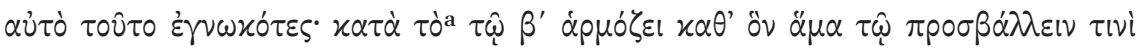

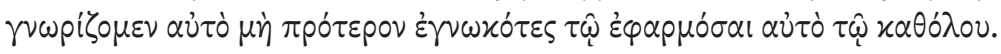


Schol. e Phlp. in APo CAG XIII 3, p. 16,30-17,4 excerpt., i. l. pos. et nigro atram. scr.

a $\varkappa \alpha \tau \dot{\alpha} \tau \dot{~ m i h i ~ n o n ~ l i q u e t ~}$

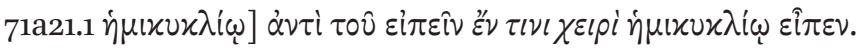

Schol. e Phlp. in APo CAG XIII 3, p. 17,14-15 excerpt., i. l. pos. et nigro atram. scr.

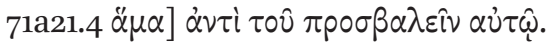

Schol. i. l. pos. et nigro atram. scr.

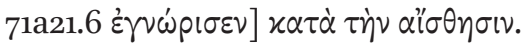

Schol. i. l. pos. et nigro atram. scr.

\section{Laurentianus 72,4}

f. 139v:

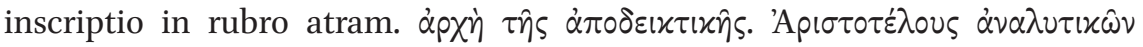

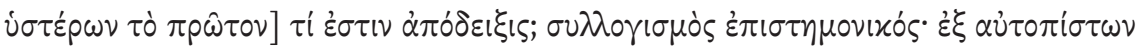

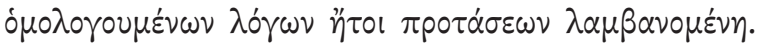

Schol. e loco Philoponeo CAG XIII 3, p. 3,1-2 excerpt. anteced. Aristotelis APo. Similiter et schol. in 71a1.1-2.3 cod. Princeton MS. 173 docet.

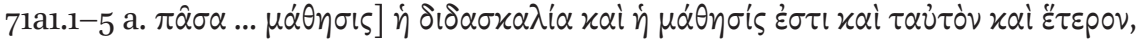

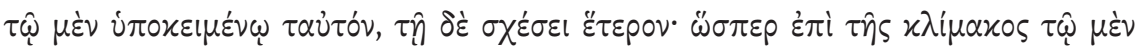

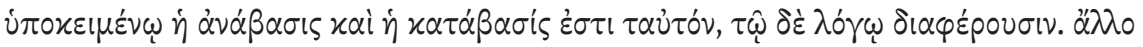

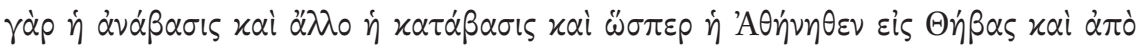

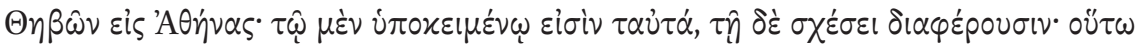

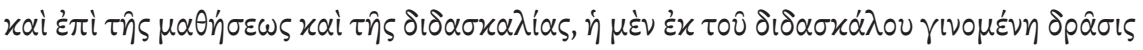

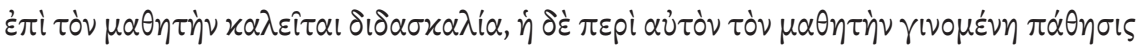
$\mu \alpha \dot{\theta} \eta \sigma \iota \varsigma \pi \rho \circ \sigma \alpha \gamma \circ \rho \varepsilon \varepsilon^{\varepsilon} \varepsilon \alpha \iota$.

Schol. e Phlp. in APo CAG XIII 3, p. 4,16-22 excerpt. anteced. Aristotelis APo. Fere eadem schol. in 71a1.2 cod. Princeton MS. 173 tradit.

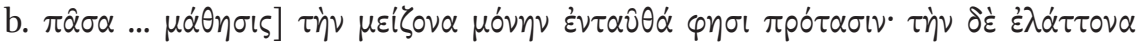
$\pi \alpha \rho \varepsilon i \alpha \sigma \varepsilon$.

Schol. s. l. pos. Similia et in schol. in 71a1.6 b. quod in cod. Princeton MS 173 legitur. 


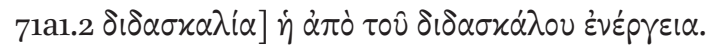

Schol. e loco Philoponeo CAG XIII 3, p. 4,20-21 ort., s. l. scr.

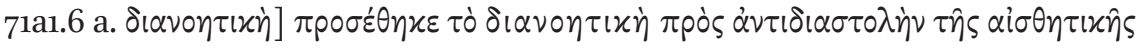

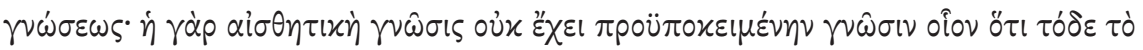

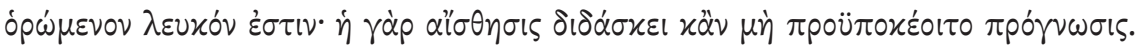

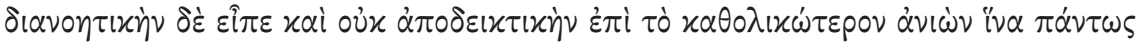

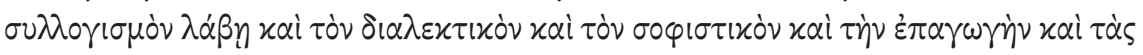

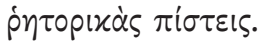

Schol. e Phlp. in APo CAg XIII 3, p. 4,30-5,6 excerpt., in mg. int. pos. Fere eadem in schol. in 71a1.6 a. cod. Princeton MS. 173 leguntur.

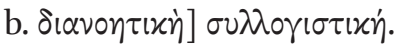

Schol. s. l. pos.

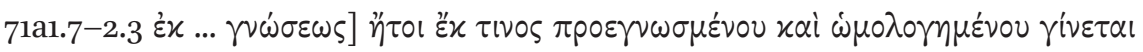
$\pi \rho \alpha ́ \gamma \mu \alpha \tau o \varsigma$.

Schol. e loco Philoponeo CAG XIII 3, p. 4,14 ort., s. l. pos. Eadem et in schol. in 71a1.8 b. cod. Princeton MS. 173 .

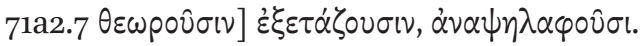

Schol. i. l. pos., idem ac schol. in 71a2.6 cod. Princeton MS. 173 .

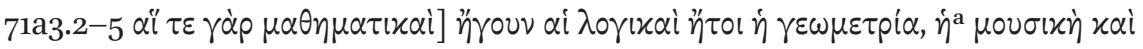
$\dot{\eta} \dot{\alpha} \rho \imath \theta \mu \eta \tau i x \dot{\eta}$.

Schol. i. l. pos., simil. schol. in 71a2.2-5 cod. Princeton MS. 173

a $\dot{\eta}$ fere evanidum

\section{$3 \quad$ Appendice I}

È dato di rintracciare i medesimi scoli in manoscritti diversi? E, di conseguenza, è possibile ricostruire corpora di scoli? In base ai sondaggi finora effettuati 
nei capitoli 1, 27 e 28 di Analitici posteriori 1, si può affermare che il Barocci 177 (metà del XIII sec.) e il Laurentianus 72,4 (fine del XIII / primo quarto del XIV sec.), nonché il Marcianus Gr. 202 e il Princetonensis MS 173, entrambi della fine del XIII sec. contengono i medesimi scoli. La tabella che segue nell'Appendice I consente di visualizzare i dati relativi al capitolo 1 dal primo libro degli Analitici posteriori in quattordici dei diciassette manoscritti, escludendo il Vaticanus Gr. 241, il Laurentianus 72,3 e il Laurentianus 72,4, i cui scoli sono stati qui sopra editi integralmente.

\section{4}

\section{Appendici II e III}

Si pubblicano infine nelle Appendici II e III due note relative a Psello lettore dell'Organon e alla Praefatio alla traduzione latina di Ermolao Barbaro della Parafrasi agli Analitici posteriori di Temistio, che consentono di gettare uno sguardo sulla tradizione esegetica relativa agli Analitici posteriori di età bizantina e umanistica.

\section{Appendice I}

\section{Mappatura degli scoli ad Aristot., Analytica Posteriora A 1 (71a17-24)}

- Ambrosianus L 93 sup.

- Vaticanus Urbinas Gr. 35

- Vaticanus Barberinianus Gr. 87

- Laurentianus 72,5

- Marcianus Gr. 201

- Parisinus Coislinianus 330

- Laurentianus CS 192

- Vaticanus Gr. 244

- Guelferbytanus 24 Gud. Gr.

- Barocci 177

- Monacensis Gr. 222

- Princeton. MS. 173

- Vaticanus Barberinianus Gr. 164

- Marcianus Gr. 202
(IX sec.)

(Ix/X sec.)

(x sec.)

(seconda metà del X sec.)

(954)

(XI sec.)

(seconda metà/ultimo quarto del XII sec.)

(XIII sec.)

(XIII sec.)

(metà del XIII sec.)

(fine del XIII sec.)

(fine del XiII sec.)

(fine del XIII sec.)

(fine del XIII sec.) 


\begin{tabular}{|c|c|c|c|c|c|c|c|}
\hline $\begin{array}{l}\text { Riferimento } \\
\text { all'edizione } \\
\text { di Bekker }\end{array}$ & $\begin{array}{l}\text { Edizione } \\
\text { OCT } \\
1964\end{array}$ & $\begin{array}{l}\text { Ambr. L } \\
93 \text { sup. } \\
\text { (fol. 19ov) }\end{array}$ & $\begin{array}{l}\text { Urb. Gr. } 35 \\
\text { (fol. 193v) }\end{array}$ & $\begin{array}{l}\text { Vat. Barber. } \\
\text { Gr. } 87 \\
\text { (fol. 121r) }\end{array}$ & $\begin{array}{l}\text { Laur. } 72,5 \\
\text { (fol. 153r) }\end{array}$ & $\begin{array}{l}\text { Marc. } \\
\text { Gr. } 201 \\
\text { (fol. } 85 \mathrm{v} \text { ) }\end{array}$ & $\begin{array}{l}\text { Coisl. } 330 \\
\text { (fol. 15or) }\end{array}$ \\
\hline 71a17.01-18.05 & 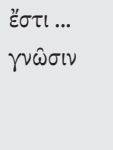 & & & $\begin{array}{l}\pi \alpha \rho \alpha \dot{\delta} \varepsilon l \gamma \mu \alpha \\
\varepsilon ૈ \sigma \tau \omega \lambda i \theta_{\circ \nu} \\
\mu \alpha \gamma \nu \dot{\tau} \tau l \\
\delta \circ \varsigma^{\mathrm{a}}\end{array}$ & & & \\
\hline $71 \mathrm{a} 17.01$ & है $\sigma \tau \iota$ & & & & & 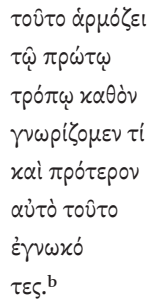 & \\
\hline
\end{tabular}

\begin{tabular}{|c|c|}
\hline 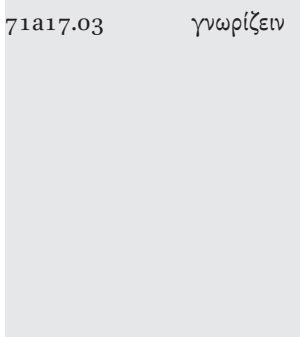 & 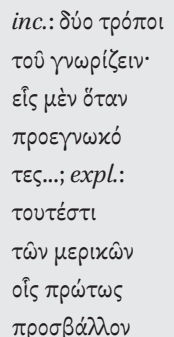 \\
\hline
\end{tabular}




\begin{tabular}{|c|c|c|c|c|c|c|c|}
\hline $\begin{array}{l}\text { Laur. CS } 192 \\
\text { (fol. } 73^{r} \text { ) }\end{array}$ & $\begin{array}{l}\text { Vat. Gr. } 244 \\
\text { (fol. 302v) }\end{array}$ & $\begin{array}{l}\text { Guelf. } 24 \\
\text { Gud. Gr. } \\
\text { (fol. } 85 \text { r) }\end{array}$ & $\begin{array}{l}\text { Barocci } \\
177 \\
\text { (fol. } 83 \mathrm{v} \text { ) }\end{array}$ & $\begin{array}{l}\text { Monacensis } \\
\text { Gr. } 222 \\
\text { (fol. 142r) }\end{array}$ & $\begin{array}{l}\text { Princeton } \\
\text { MS. } 173 \\
\text { (fol. } 78 \mathrm{r} \text { ) }\end{array}$ & $\begin{array}{l}\text { Vat. Barber. } \\
\text { Gr. } 164 \\
\text { (fol. 122r) }\end{array}$ & $\begin{array}{l}\text { Marcianus } \\
\text { Gr. } 202 \\
\text { (fol. 258v) }\end{array}$ \\
\hline
\end{tabular}

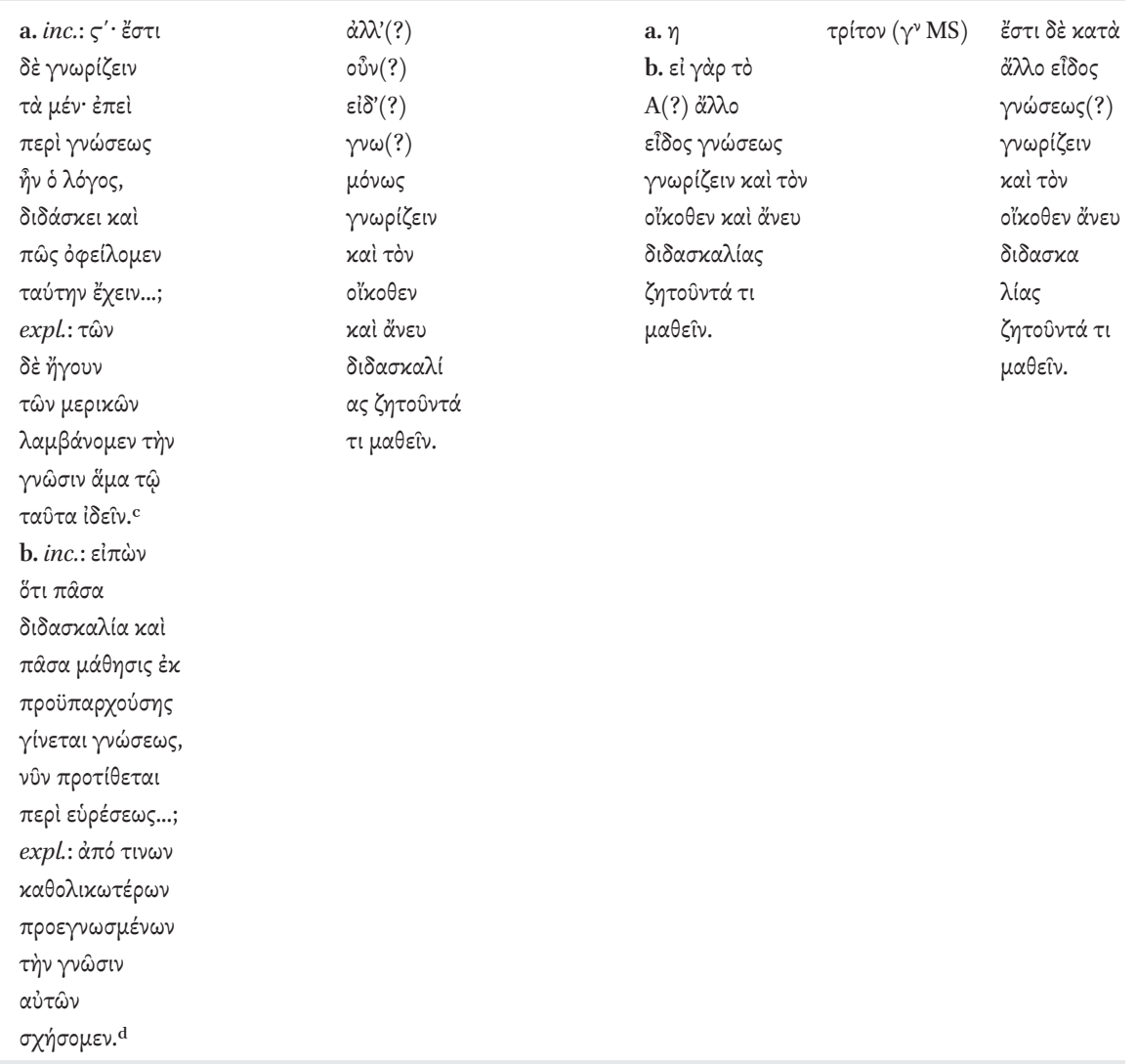

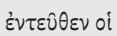

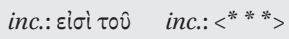

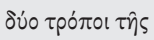

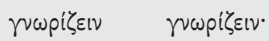

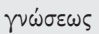

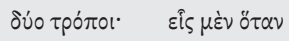

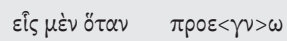

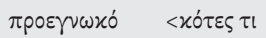

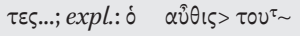

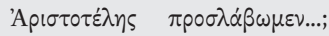

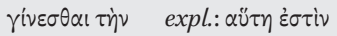

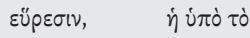


TABLE (cont.)

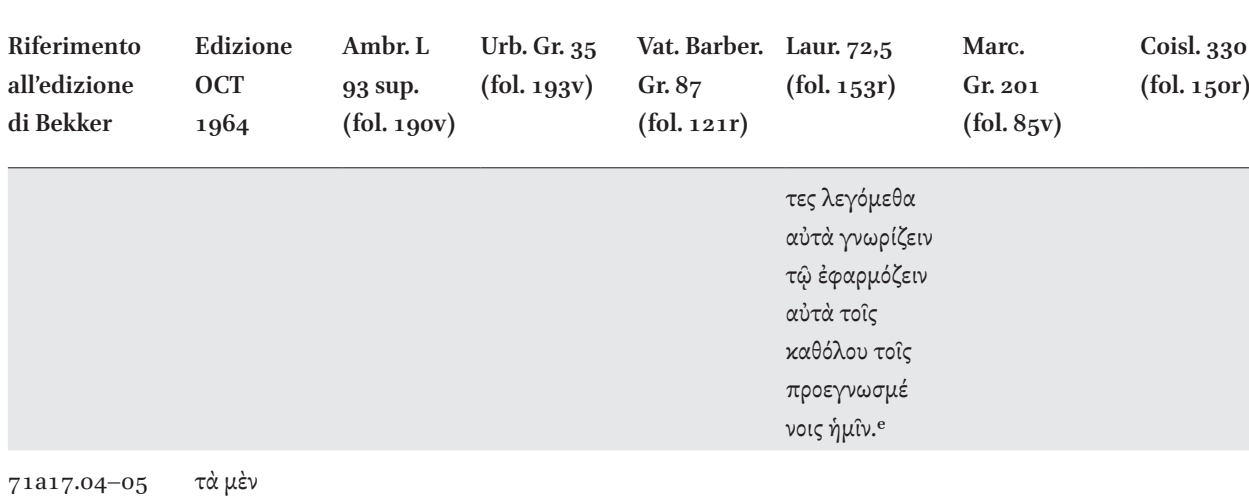

\begin{tabular}{|c|c|c|}
\hline $71 \mathrm{a} 17.07$ & $\gamma \nu \omega \rho i \sigma \alpha \nu \tau \alpha$ & \\
\hline \multirow[t]{9}{*}{$71 \mathrm{a} 17.08$} & $\tau \hat{\omega} \nu$ & $\tau \hat{\omega} \nu \mu \varepsilon \rho i x \hat{\omega} \nu$. \\
\hline & & $\tau \dot{\alpha} \gamma \dot{\alpha} \rho \mu \varepsilon \rho i x \dot{\alpha}$ \\
\hline & & 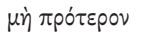 \\
\hline & & 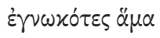 \\
\hline & & $\tau \hat{\omega} \pi \rho \circ \sigma \beta \alpha \lambda \varepsilon \hat{\imath} \nu$ \\
\hline & & $\tau i v i \gamma \nu \omega p^{\prime \zeta \zeta} \mu \varepsilon v$ \\
\hline & & $\varepsilon \dot{\varepsilon} \varphi \rho \rho \mu ́ \sigma \alpha \nu \tau \varepsilon \varsigma$ \\
\hline & & $\alpha \hat{\tau} \tau \dot{~} \tau \hat{\omega}$ \\
\hline & & $x \alpha \theta 0 ́ \lambda$ ${ }^{i}$ \\
\hline
\end{tabular}

\begin{tabular}{|c|c|c|}
\hline $71 \mathrm{a} 18.02$ & $\ddot{\alpha} \mu \alpha$ & \\
\hline $71 \mathrm{a} 18.03$ & $\lambda \alpha \mu \beta \alpha^{\prime} \nu \circ \nu \tau \alpha$ & 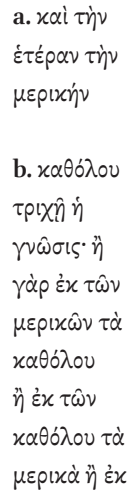 \\
\hline
\end{tabular}




\begin{tabular}{|c|c|c|c|c|c|c|c|}
\hline $\begin{array}{l}\text { Laur. CS } 192 \\
\text { (fol. } 73 \text { r) }\end{array}$ & $\begin{array}{l}\text { Vat. Gr. } 244 \\
\text { (fol. } 302 v \text { ) }\end{array}$ & $\begin{array}{l}\text { Guelf. } 24 \\
\text { Gud. Gr. } \\
\text { (fol. } 85 \text { r) }\end{array}$ & $\begin{array}{l}\text { Barocci } \\
177 \\
(\text { fol. } 83 v)\end{array}$ & $\begin{array}{l}\text { Monacensis } \\
\text { Gr. } 222 \\
\text { (fol. 142r) }\end{array}$ & $\begin{array}{l}\text { Princeton } \\
\text { MS. } 173 \\
\text { (fol. } 78 \mathrm{r} \text { ) }\end{array}$ & $\begin{array}{l}\text { Vat. Barber. } \\
\text { Gr. } 164 \\
\text { (fol. 122r) }\end{array}$ & $\begin{array}{l}\text { Marcianus } \\
\text { Gr. } 202 \\
\text { (fol. 258v) }\end{array}$ \\
\hline & & & & 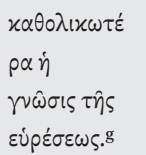 & 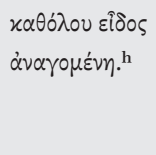 & & \\
\hline & & 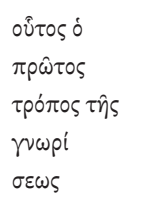 & $\tau \dot{\alpha} x \alpha \theta \dot{\partial} \lambda \circ v$ & & & & $\begin{array}{l}\tau \dot{\alpha} \mu \varepsilon \dot{\nu} \nu \\
\kappa \alpha \theta \dot{\lambda}{ }^{\circ} 0 v\end{array}$ \\
\hline & & & & & & $\tau \dot{\alpha} x \alpha \theta \dot{\lambda} \lambda \circ v$ & \\
\hline$\tau \dot{\alpha} \mu \varepsilon p l \varkappa \alpha \dot{~}$ & 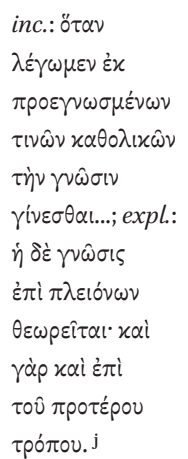 & 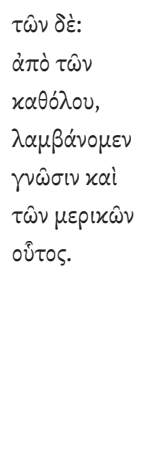 & 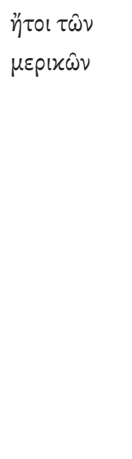 & & $\tau \hat{\omega} \nu \mu \varepsilon p เ x \hat{\omega} \nu$ & $\tau \grave{\alpha} \mu \varepsilon p l x \grave{\alpha}$ & $\begin{array}{l}\tau \hat{\omega} \nu \delta \dot{\varepsilon} \\
\mu \varepsilon p i x \hat{\omega} \nu\end{array}$ \\
\hline
\end{tabular}

$\tau \dot{\alpha} \delta \dot{\varepsilon}$

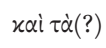

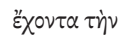

$x \alpha \theta \dot{\lambda}$ ov

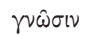

$\tau \hat{\omega} \pi \rho \circ \varepsilon \gamma \nu \omega$

$\sigma \mu \varepsilon \dot{\varepsilon} \omega \tau \hat{\imath} \varsigma$

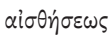

$\tau \hat{\omega} \pi \rho \circ \sigma u \lambda \gamma_{\gamma}$

$\sigma \mu \hat{\omega} \tau \hat{\eta} \varsigma$

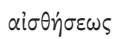


TABLE (cont.)

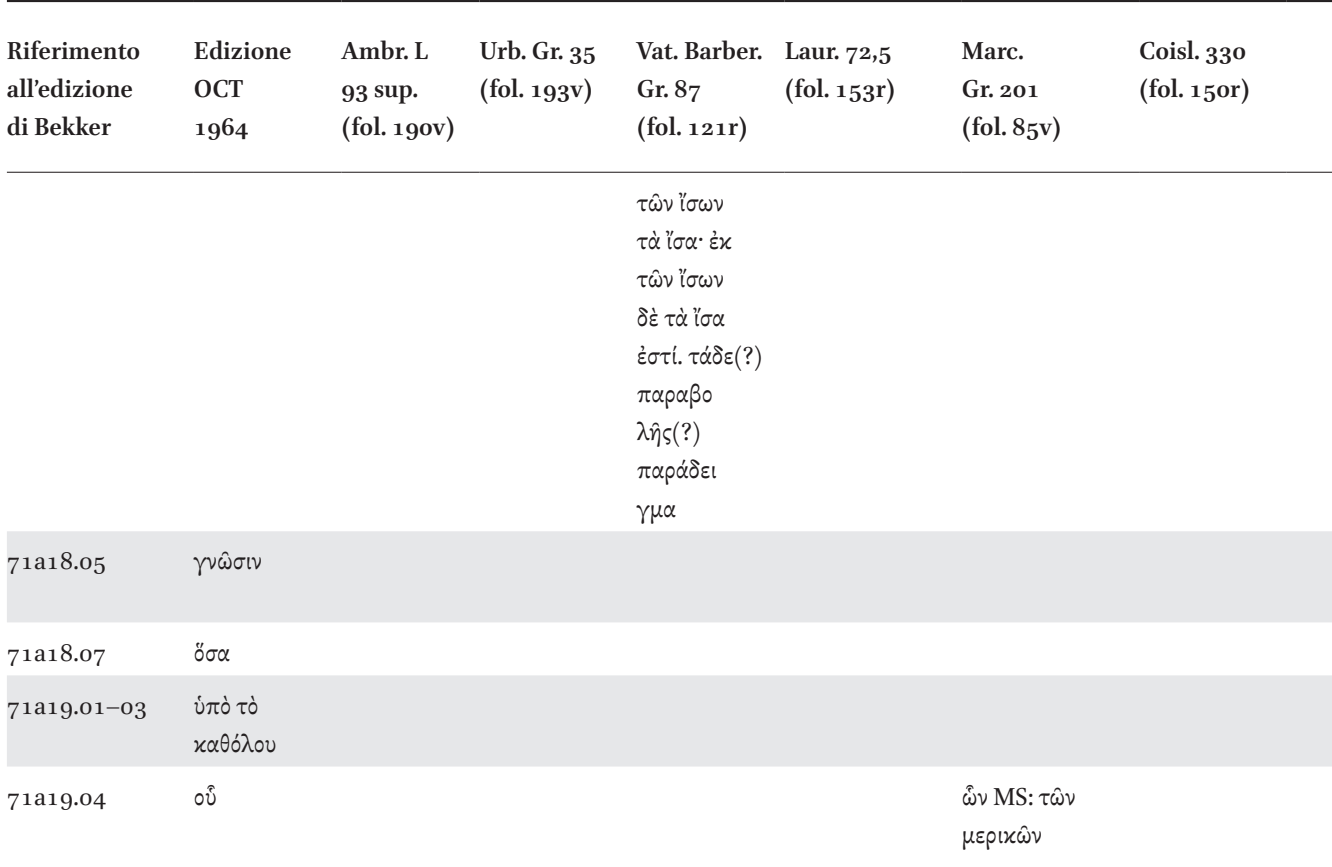

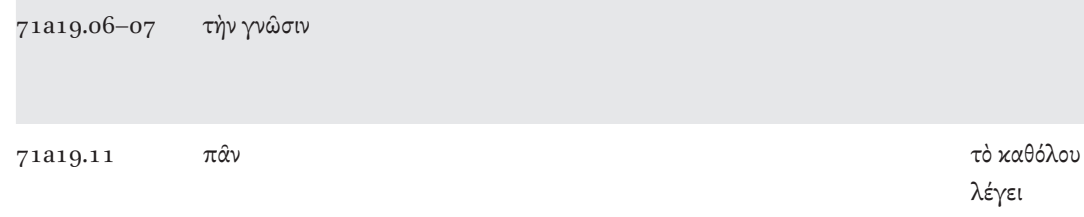




$\begin{array}{llllllll}\begin{array}{lllll}\text { Laur. CS 192 } \\ \text { (fol. 73r) }\end{array} & \text { (fol. Go2v) } & \text { Gud. Gr. } & \text { 177 } & \text { Gr. 222 } & \text { MS. 173 } & \text { Gr. 164 } & \text { Gr. 202 } \\ & & \text { (fol. 85r) } & \text { (fol. 83v) } & \text { (fol. 142r) } & \text { (fol. 78r) } & \text { (fol. 122r) } & \text { (fol. 258v) }\end{array}$

$\begin{array}{lll}\pi \circ \hat{o} \alpha \delta \dot{\eta} & \pi \circ \hat{\imath} \alpha \delta \dot{\eta} \tau \alpha \hat{\tau} \tau \alpha & \pi \circ \hat{\imath} \alpha \delta \dot{\eta} \\ \tau \alpha \hat{\tau} \tau \alpha & \tau \alpha \hat{\tau} \tau \alpha\end{array}$

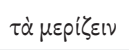

\begin{tabular}{|c|c|c|c|c|c|c|}
\hline \multirow[t]{2}{*}{$\mu \varepsilon \rho i x \grave{\alpha}$} & & & $\tau \dot{\alpha} \mu \varepsilon p i x \dot{\alpha}$ & $\tau \dot{\alpha} \mu \varepsilon p(x \dot{\alpha} x \alpha \grave{i}$ & $\ddot{\eta r \gamma o u v} \tau \dot{\alpha}$ & $\tau \dot{\alpha} \mu \varepsilon p i x \dot{\alpha}$ \\
\hline & 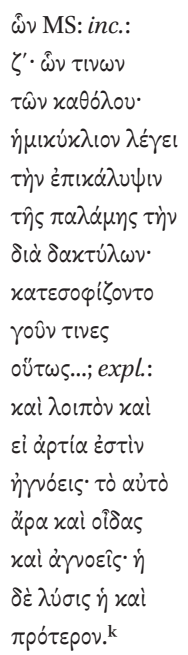 & $\begin{array}{l}\hat{\omega} v \text { MS: } \tau \hat{\omega} \nu \\
\kappa \alpha \theta \dot{\lambda} \text { ov }\end{array}$ & $\begin{array}{l}\hat{\omega} v \text { MS: } \tau \hat{\omega} v \\
\varkappa \alpha \theta \dot{\lambda} \lambda \circ v\end{array}$ & $\begin{array}{l}\hat{\omega} v \text { MS: } \tau \hat{\omega} \nu \\
\varkappa \alpha \theta \dot{\lambda} \lambda \circ v\end{array}$ & & $\begin{array}{l}\hat{\omega} \nu \mathrm{MS}: \tau \hat{\omega} \nu \\
\kappa \alpha \theta \dot{\lambda} \lambda \text { ov }\end{array}$ \\
\hline $\begin{array}{l}x \alpha \tau \dot{\alpha}(?) \\
\tau \dot{\eta} \nu(?) \ldots\end{array}$ & & & 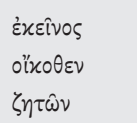 & 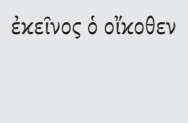 & $\begin{array}{l}\ddot{\alpha} \mu \alpha \tau \hat{\eta} \\
\alpha i \sigma \theta \dot{\eta} \sigma \varepsilon l\end{array}$ & 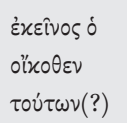 \\
\hline
\end{tabular}


TABLE (cont.)

\begin{tabular}{|c|c|c|c|c|c|c|c|}
\hline $\begin{array}{l}\text { Riferimento } \\
\text { all'edizione } \\
\text { di Bekker }\end{array}$ & $\begin{array}{l}\text { Edizione } \\
\text { OCT } \\
1964\end{array}$ & $\begin{array}{l}\text { Ambr. L } \\
93 \text { sup. } \\
\text { (fol. 19ov) }\end{array}$ & $\begin{array}{l}\text { Urb. Gr. } 35 \\
\text { (fol. 193v) }\end{array}$ & $\begin{array}{l}\text { Vat. Barber. } \\
\text { Gr. } 87 \\
\text { (fol. 121r) }\end{array}$ & $\begin{array}{l}\text { Laur. 72,5 } \\
\text { (fol. 153r) }\end{array}$ & $\begin{array}{l}\text { Marc. } \\
\text { Gr. } 201 \\
\text { (fol. } 85 \mathrm{v} \text { ) }\end{array}$ & $\begin{array}{l}\text { Coisl. } 330 \\
\text { (fol. 15or) }\end{array}$ \\
\hline 71a19.11-20.04 & 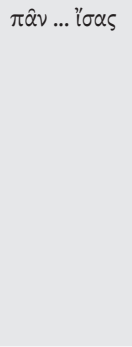 & 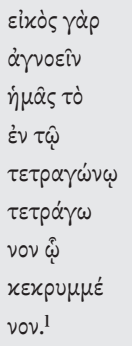 & & & & & \\
\hline $71 \mathrm{a} 20.05$ & 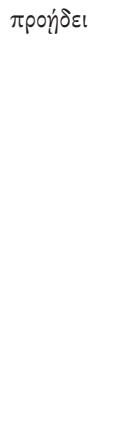 & & & & 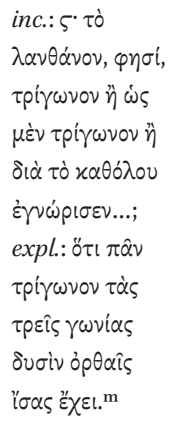 & & \\
\hline $71 \mathrm{a} 20.08$ & $\tau o ́ \delta \varepsilon$ & & & & & $\begin{array}{l}\tau \dot{\mu} \mu \varepsilon p \text { เxòv } \\
\lambda \varepsilon \dot{\varepsilon} \gamma \varepsilon \iota\end{array}$ & \\
\hline $71 \mathrm{a} 20.10$ & $\varepsilon \nu$ & & & & & 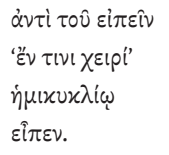 & \\
\hline $71 \mathrm{a} 20.11-21.01$ & $\begin{array}{l}\tau \hat{\omega} \\
\dot{\eta} \mu i x \cup x \lambda i \omega\end{array}$ & & 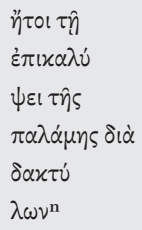 & & & & \\
\hline $71 \mathrm{a} 21.04$ & $\ddot{\alpha} \mu \alpha$ & & & & & 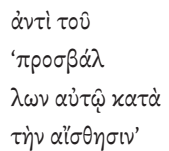 & \\
\hline
\end{tabular}




\begin{tabular}{|c|c|c|c|c|c|c|c|}
\hline $\begin{array}{l}\text { Laur. CS } 192 \\
\text { (fol. } 73^{r} \text { ) }\end{array}$ & $\begin{array}{l}\text { Vat. Gr. } 244 \\
\text { (fol. 302v) }\end{array}$ & $\begin{array}{l}\text { Guelf. } 24 \\
\text { Gud. Gr. } \\
\text { (fol. } 85 \text { r) }\end{array}$ & $\begin{array}{l}\text { Barocci } \\
177 \\
\text { (fol. } 83 \mathrm{v} \text { ) }\end{array}$ & $\begin{array}{l}\text { Monacensis } \\
\text { Gr. } 222 \\
\text { (fol. 142r) }\end{array}$ & $\begin{array}{l}\text { Princeton } \\
\text { MS. 173 } \\
\text { (fol. } 78 \mathrm{r} \text { ) }\end{array}$ & $\begin{array}{l}\text { Vat. Barber. } \\
\text { Gr. } 164 \\
\text { (fol. 122r) }\end{array}$ & $\begin{array}{l}\text { Marcianus } \\
\text { Gr. } 202 \\
\text { (fol. 258v) }\end{array}$ \\
\hline
\end{tabular}


TABLE (cont.)

\begin{tabular}{|c|c|c|c|c|c|c|c|}
\hline $\begin{array}{l}\text { Riferimento } \\
\text { all'edizione } \\
\text { di Bekker }\end{array}$ & $\begin{array}{l}\text { Edizione } \\
\text { OCT } \\
1964\end{array}$ & $\begin{array}{l}\text { Ambr. L } \\
93 \text { sup. } \\
\text { (fol. 19ov) }\end{array}$ & $\begin{array}{l}\text { Urb. Gr. } 35 \\
\text { (fol. 193v) }\end{array}$ & $\begin{array}{l}\text { Vat. Barber. } \\
\text { Gr. } 87 \\
\text { (fol. 121r) }\end{array}$ & $\begin{array}{l}\text { Laur. 72,5 } \\
\text { (fol. 153r) }\end{array}$ & $\begin{array}{l}\text { Marc. } \\
\text { Gr. } 201 \\
\text { (fol. 85v) }\end{array}$ & $\begin{array}{l}\text { Coisl. } 330 \\
\text { (fol. 15or) }\end{array}$ \\
\hline $71 \mathrm{a} 21.05$ & 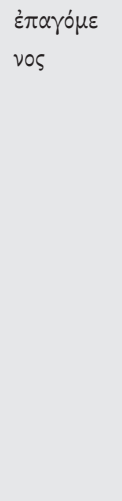 & & $\begin{array}{l}\theta \varepsilon \alpha \sigma \alpha \dot{\alpha} \mu \varepsilon \\
\nu \circ \varsigma^{\circ}\end{array}$ & $\begin{array}{l}\text { a. } \pi \rho \circ \sigma \beta \alpha \\
\lambda \omega^{\prime} \nu, \\
\theta \varepsilon \alpha \sigma \dot{\alpha} \mu \varepsilon \\
\nu \circ \varsigma^{p} \\
\text { b. } \delta \imath^{\prime} \\
\varepsilon \dot{\tau} \pi \gamma \omega \gamma \eta \hat{\gamma} \varsigma \\
\gamma \nu \omega \rho i \zeta \varepsilon l \tau \dot{\alpha} \\
\mu \varepsilon \rho l \\
\chi \dot{\alpha}^{q}\end{array}$ & 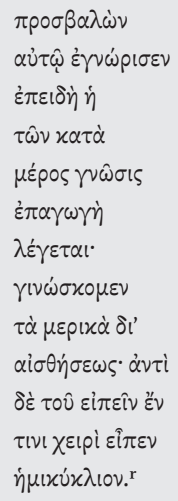 & & $\begin{array}{l}\alpha i \sigma \theta \dot{\sigma} \sigma \varepsilon l \\
\pi \rho \circ \sigma \beta \alpha \\
\lambda \omega \dot{\omega} \nu\end{array}$ \\
\hline $71 \mathrm{a} 21.07-23.03$ & 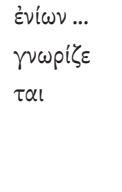 & & & 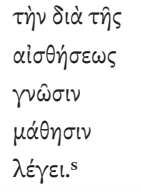 & & & \\
\hline $71 \mathrm{a} 21.07$ & 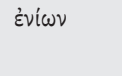 & $\begin{array}{l}\alpha \nu \tau i \tau \hat{\omega} \nu \\
\mu \varepsilon \rho i x \omega \hat{\omega} v^{t}\end{array}$ & $\begin{array}{l}\eta ँ \tau o l \tau \hat{\omega} \nu \\
\mu \varepsilon \rho เ x \omega \hat{\omega} \nu\end{array}$ & & & $\tau \hat{\omega} \nu \mu \varepsilon \rho \iota x \omega \hat{\omega} \nu$ & \\
\hline $71 \mathrm{a} 22.02$ & 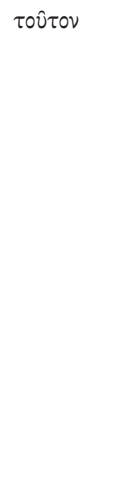 & & 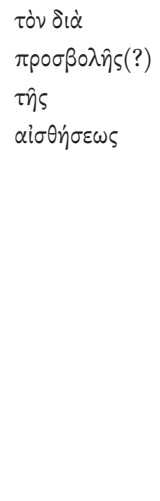 & $\begin{array}{l}\tau \dot{\nu} \nu \delta i \dot{\alpha} \tau \hat{\varsigma} \varsigma \\
\dot{\varepsilon} \pi \alpha \gamma \omega \gamma \hat{\varsigma} s\end{array}$ & 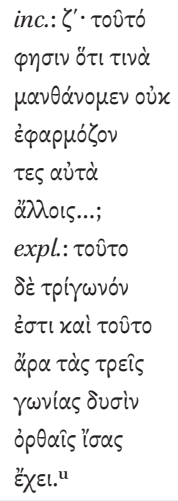 & 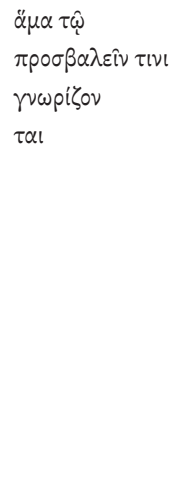 & \\
\hline $71 \mathrm{a} 22.03$ & $\tau o ̀ v$ & 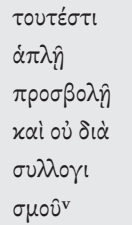 & & & & & \\
\hline
\end{tabular}




\begin{tabular}{|c|c|c|c|c|c|c|c|}
\hline $\begin{array}{l}\text { Laur. CS } 192 \\
\text { (fol. } 73^{r} \text { ) }\end{array}$ & $\begin{array}{l}\text { Vat. Gr. } 244 \\
\text { (fol. 302v) }\end{array}$ & $\begin{array}{l}\text { Guelf. } 24 \\
\text { Gud. Gr. } \\
\text { (fol. } 85 \text { r) }\end{array}$ & $\begin{array}{l}\text { Barocci } \\
177 \\
\text { (fol. } 83 \mathrm{v} \text { ) }\end{array}$ & $\begin{array}{l}\text { Monacensis } \\
\text { Gr. } 222 \\
\text { (fol. 142r) }\end{array}$ & $\begin{array}{l}\text { Princeton } \\
\text { MS. 173 } \\
\text { (fol. } 78 \mathrm{r} \text { ) }\end{array}$ & $\begin{array}{l}\text { Vat. Barber. } \\
\text { Gr. } 164 \\
\text { (fol. 122r) }\end{array}$ & $\begin{array}{l}\text { Marcianus } \\
\text { Gr. } 202 \\
\text { (fol. 258v) }\end{array}$ \\
\hline
\end{tabular}

Idem 
TABLE (cont.)

\begin{tabular}{|c|c|c|c|c|c|c|c|}
\hline $\begin{array}{l}\text { Riferimento } \\
\text { all'edizione } \\
\text { di Bekker }\end{array}$ & $\begin{array}{l}\text { Edizione } \\
\text { OCT } \\
\mathbf{1 9 6 4}\end{array}$ & $\begin{array}{l}\text { Ambr. L } \\
93 \text { sup. } \\
\text { (fol. 19ov) }\end{array}$ & $\begin{array}{l}\text { Urb. Gr. } 35 \\
\text { (fol. 193v) }\end{array}$ & $\begin{array}{l}\text { Vat. Barber. } \\
\text { Gr. } 87 \\
\text { (fol. 121r) }\end{array}$ & $\begin{array}{l}\text { Laur. 72,5 } \\
\text { (fol. 153r) }\end{array}$ & $\begin{array}{l}\text { Marc. } \\
\text { Gr. } 201 \\
(\text { fol. } 85 v)\end{array}$ & $\begin{array}{l}\text { Coisl. } 33^{\circ} \\
\text { (fol. 15or) }\end{array}$ \\
\hline $71 \mathrm{a} 22.04$ & 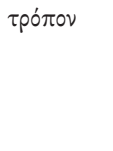 & & & & $\begin{array}{l}\tau \dot{\eta} \nu \delta ı \dot{\alpha} \tau \hat{\eta} \varsigma \\
\alpha \dot{i} \sigma \dot{\eta} \sigma \varepsilon \omega \varsigma \\
\gamma \nu \hat{\omega} \sigma \iota \nu \mu \dot{\alpha} \theta \eta \sigma \iota \nu \\
\varepsilon \hat{i} \pi \varepsilon .\end{array}$ & & \\
\hline $71 \mathrm{a22.06}$ & $\mu \alpha \dot{\theta} \eta \sigma ı s$ & & & & & 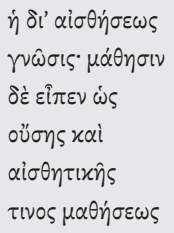 & \\
\hline $71 \mathrm{a} 22.10$ & $\delta i \dot{\alpha}$ & 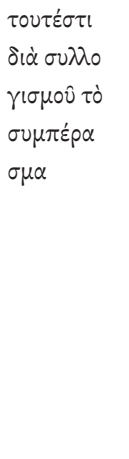 & & 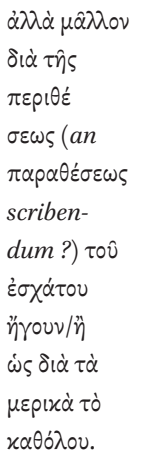 & & & \\
\hline $71 \mathrm{a} 22.12$ & $\mu \varepsilon \dot{\sigma} \sigma \nu$ & & & & & 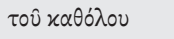 & $\dot{\alpha} \pi \circ \delta \varepsilon \mid \xi \varepsilon \omega \varsigma$ \\
\hline $71 \mathrm{a} 23.02$ & है$\sigma \chi \alpha \tau o \nu$ & & 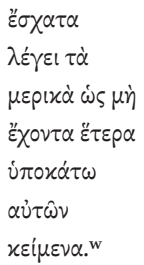 & & & 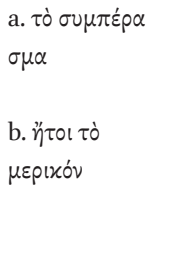 & \\
\hline $71 \mathrm{a} 23.04$ & 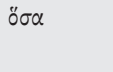 & & & 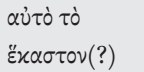 & & & \\
\hline
\end{tabular}




$\begin{array}{llllllll}\begin{array}{l}\text { Laur. CS 192 Vat. Gr. 244 } \\ \text { (fol. 73r) }\end{array} & \text { Guelf. 24 } & \text { Barocci } & \text { Monacensis } & \text { Princeton } & \text { Vat. Barber. } & \text { Marcianus } \\ & & \text { Gud. Gr. } & 177 & \text { Gr. 222 } & \text { MS. 173 } & \text { Gr. 164 } & \text { Gr. 202 } \\ & & \text { (fol. 85r) } & \text { (fol. 83v) } & \text { (fol. 142r) } & \text { (fol. 78r) } & \text { (fol. 122r) } & \text { (fol. 258v) }\end{array}$


TABLE (cont.)

\begin{tabular}{|c|c|c|c|c|c|c|c|}
\hline $\begin{array}{l}\text { Riferimento } \\
\text { all'edizione } \\
\text { di Bekker }\end{array}$ & $\begin{array}{l}\text { Edizione } \\
\text { OCT } \\
1964\end{array}$ & $\begin{array}{l}\text { Ambr. L } \\
93 \text { sup. } \\
\text { (fol. 19ov) }\end{array}$ & $\begin{array}{l}\text { Urb. Gr. } 35 \\
\text { (fol. 193v) }\end{array}$ & $\begin{array}{l}\text { Vat. Barber. } \\
\text { Gr. } 87 \\
\text { (fol. 121r) }\end{array}$ & $\begin{array}{l}\text { Laur. 72,5 } \\
\text { (fol. 153r) }\end{array}$ & $\begin{array}{l}\text { Marc. } \\
\text { Gr. } 201 \\
\text { (fol. } 85 \mathrm{v} \text { ) }\end{array}$ & $\begin{array}{l}\text { Coisl. } 330 \\
\text { (fol. 15or) }\end{array}$ \\
\hline $71 \mathrm{a} 24.03$ & $\mu \grave{\eta}$ & 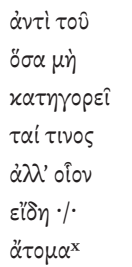 & & & & & \\
\hline $71 \mathrm{a} 24.05$ & 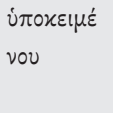 & & & & & 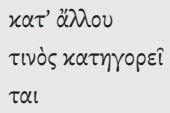 & \\
\hline
\end{tabular}

a La nota è vergata in scriptura semiuncialis, cfr. Capocci 1958: 118. Lo scoliasta si riferisce all'esempio del magnete riportato anche da Phlp., in APo CAG XIII 3, p. 12,24-29.

b Estratto da Phlp., in APo CAG XIII 3, p. 16,30-17,1.

c Estratto da Eustr., in APo CAG XXI 1, p. XI,24-31.

d Cfr. Phlp., in APo CAG XIII 3, p. 12,6-13,4 e anche Eustr., in APo CAG XXI 1, p. XI,32-XII,19.

e Estratto da Phlp., in APo CAG XIII 3, p. 12,16-23 e 17,5-8.

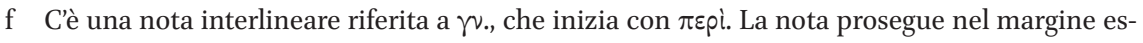
terno. La bassa qualità dell'immagine a mia disposizione mi permette di riconoscere soltanto

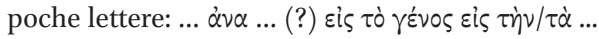

g Estratto da Phlp., in APo CAG XIII 3, p. 12,16-24 + 13,1-4.

h Estratto da Phlp., in APo CAG XIII 3, p. 12,16-29.

i Estratto da Phlp., in APo CAG XIII 3, p. 17,3-4.

j Estratto da Phlp., in APo CAG XIII 3, p. 13,29-14,5.

$\mathrm{k}$ Estratto da Eustr., in APo CAG XXI 1, p. XII, 25-36. 


\begin{tabular}{|c|c|c|c|c|c|c|c|}
\hline $\begin{array}{l}\text { Laur. CS } 192 \\
\text { (fol. } 73 \text { r) }\end{array}$ & $\begin{array}{l}\text { Vat. Gr. } 244 \\
\text { (fol. 302v) }\end{array}$ & $\begin{array}{l}\text { Guelf. } 24 \\
\text { Gud. Gr. } \\
\text { (fol. 85r) }\end{array}$ & $\begin{array}{l}\text { Barocci } \\
177 \\
\text { (fol. 83v) }\end{array}$ & $\begin{array}{l}\text { Monacensis } \\
\text { Gr. } 222 \\
\text { (fol. 142r) }\end{array}$ & $\begin{array}{l}\text { Princeton } \\
\text { MS. } 173 \\
\text { (fol. } 78 \mathrm{r} \text { ) }\end{array}$ & $\begin{array}{l}\text { Vat. Barber. } \\
\text { Gr. } 164 \\
\text { (fol. 122r) }\end{array}$ & $\begin{array}{l}\text { Marcianus } \\
\text { Gr. } 202 \\
\text { (fol. 258v) }\end{array}$ \\
\hline
\end{tabular}

1 Probabilmente estratto da Phlp., in APo CAG XIII 3, p. 14,23-15,19.

$m$ Estratto da Phlp., in APo CAG XIII 3, p. 17,15-17 + 18-21.

$n$ Estratto da Eustr., in APo CAG XXI 1, p. XII, 25-26.

o Estratto da Eustr., in APo CAG XXI 1, p. XII, 368.

p Estratto da Eustr., in APo CAG XXI 1, p. XII, 36-37.

q Probabilmente estratto da Phlp., in APo CAG XIII 3, p. 17,12-14.

r Estratto da Phlp., in APo CAG XIII 3, p. 17,12-14.

s Estratto da Phlp., in APo CAG XIII 3, p. 18,7.

t Probabilmente estratto da Phlp., in APo CAG XIII 3, p. 17,28-18,1.

u Estratto da Phlp., in APo CAG XIII 3, p. 17,25-18,6.

v La nota sembra dipendere da Phlp., in APo CAG XIII 3, p. 17,12 o 17,26.

w Estratto da Eustr., in APo CAG XXI 1, p. XIII, 5-6.

x Per la glossa introdotta dal segno $\%$, nessun riferimento nel testo principale. 


\section{Appendice II}

\section{Psello lettore dell'Organon: Michele Psello, Opuscula philosophica minora (PhM, I 5 Duffy)}

Assecondando la richiesta di un suo amico, Costantino Xifilino, all'epoca co-

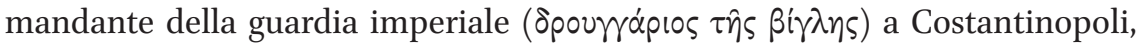

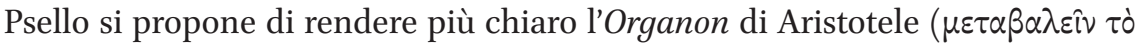

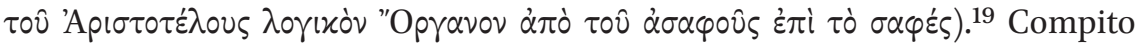
niente affatto leggero ( $\hat{\alpha} \theta \lambda \circ \varsigma \beta \alpha \rho \dot{\tau} \tau \alpha \tau \varsigma)$ ), rispetto al quale il dotto bizantino, in ossequio senz'altro alla retorica della $\tau \alpha \pi \varepsilon เ v \omega \dot{\omega} \tau \eta \varsigma^{, 20}$ ammette nelle prime linee di questo saggio (I 5) la propria inadeguatezza. Causa ne è lo scetticismo sulle proprie possibilità in campo retorico e, soprattutto, filosofico. ${ }^{21}$

'E $\pi \varepsilon i$ oûv xai 'Ap

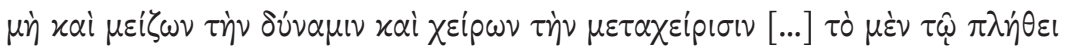

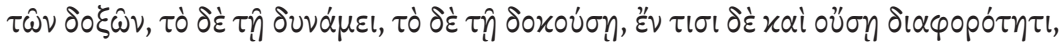

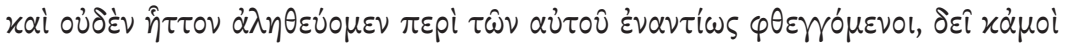

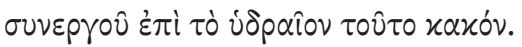

'Dato che dunque Aristotele non è affatto da meno dell'Idra che rinasce ogni volta, se non addirittura più grande in termini di potenza e più difficile da catturare, [...] vuoi per il gran numero di idee vuoi per la (loro) superiorità vuoi per la (loro) diversità, ora apparente ora in taluni casi reale, e (dato che) cionondimeno diciamo la verità quando esprimiamo un parere contrario riguardo alle sue idee, anche a me serve un assistente contro questo avversario che è come un'idra.'. ${ }^{22}$

19 Duffy 1992, 14,2-3.

20 Sulla inadeguatezza di Psello in campo filosofico ci sarebbero anche concreti riferimenti autobiografici nella Chronographia III 3 e VI 37. Cfr. anche Ierodiakonou 2002, 158.

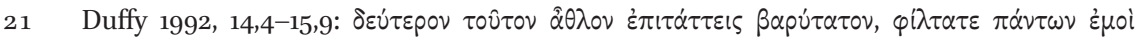

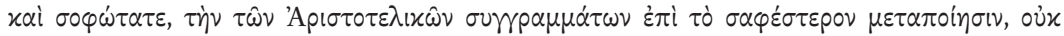

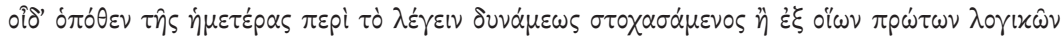

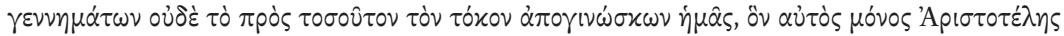

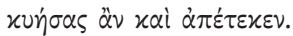

22 Duffy 1992, 16,58-17,65. Psello potrebbe aver ricavato la metafora di Ercole e l'Idra dall' $E u$ tidemo di Platone (297c1-d2). In particolare, nelle parole di PhM I 5, p. 16, 55-56 Duffy:

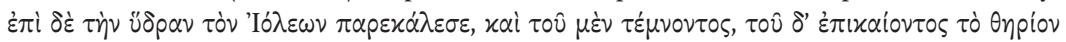

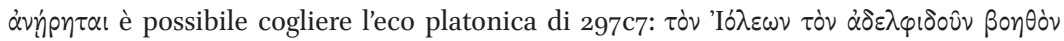
$\varepsilon \dot{\varepsilon} \varepsilon \kappa \alpha \lambda \varepsilon \dot{\varepsilon} \sigma \alpha \tau$. La metafora di Eracle e Iolao ricorre anche in una lettera del patriarca Gregorio di Cipro, il quale alla fine del secolo XIII commissionò a un team di due scribi 
Alla fine del passo qui sopra riportato, Psello allude a un altrimenti scono-

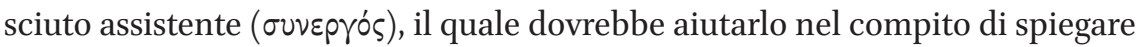
l'Organon. Chi è poi questo assistente? Frederick Lauritzen mi suggerisce per litteras la possibilità di identificarlo con Giovanni Italo in base a un riferimento al mitico Dedalo che Psello stesso fa nel paragrafo finale dell'opuscolo. ${ }^{23} \mathrm{~A}$ me non pare: Psello si rivolge in tutto l'opuscoletto a un tu, che è Costantino Xifilino; e alla fine quel tu, soprannominato Dedalo nel gioco dei vari paragoni, diventerebbe improvvisamente Italo, allievo e successore di Psello? No: Psello dice di sé che è come i garzoni di Dedalo: essi tagliano il blocco di marmo alla buona, Dedalo (Xifilino) invece digrossa, rifinisce. Psello, quindi, prepara per il suo lettore un testo, che il lettore interpreterà ancor meglio (pura adulazione del militare e understatement di sé stesso); e il lettore diventerà così come Zeus, che porta a compimento e rende bello e maturo nella sua coscia un Dioniso, feto altrimenti quasi abortito.

Vd. PhM I 5, p. 17,70-80:

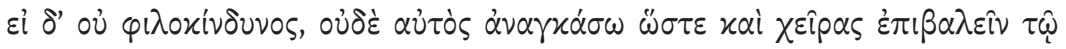
Enpíw.

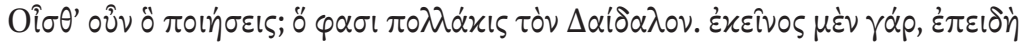

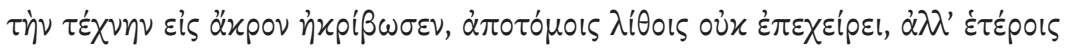

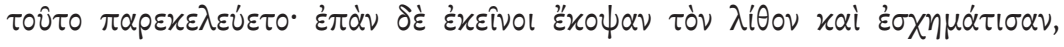

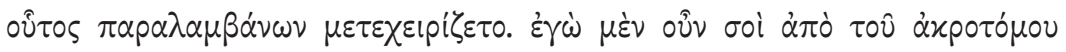

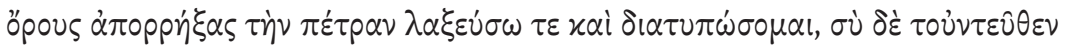

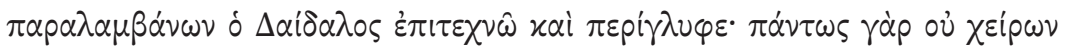

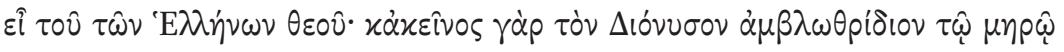

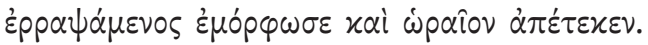

'Ma se non sei amante del pericolo, nemmeno io ti forzerò a metter pure le mani addosso alla bestia.

Sai allora ciò che devi fare? Ciò che spesso si dice facesse Dedalo. Quegli, infatti, dato che per l'appunto praticò la (propria) arte al massimo della perfezione, non metteva mano ai blocchi di pietra, ma ordinava ad altri di far questo. Ora, una volta che costoro avevano tagliato la pietra e le avevano dato forma, Dedalo la prendeva (la pietra) e la lavorava. Io,

la realizzazione di un manoscritto contenente le opere di Platone. Secondo le parole del patriarca, uno dei due scribi doveva collaborare con l'altro così come Iolao ebbe a collaborare con Ercole, cfr. Bianconi 2003, 521.

23 Cfr. Duffy 1992, 17,72. 
dunque, dopo aver staccato dalla montagna scoscesa questo macigno, te lo squadrerò e gli darò una forma; tu quindi, presolo (nelle tue mani), (novello) Dedalo, ti ingegnerai a scolpirlo tutto intorno. Infatti, non sei assolutamente da meno del dio dei Greci: anche quello, cucitosi nella coscia Dioniso, feto destinato all'aborto se non fosse stato incubato altrove, gli diede forma e lo partorì al tempo destinato'.

È interessante l'interazione che emerge da queste righe tra l'autore di un'edizione commentata dell'Organon (Psello) e il lettore (Xifilino) espressa dalla metafora di Psello-garzone e Xifilino-Dedalo. Del resto, anche noi leggiamo testi commentati da altri e, a partire dal grosso della fatica altrui, costruiamo interpretazioni più approfondite: nani sulle spalle di giganti. Dell'esegesi pselliana (una sinossi?) all'Organon di cui PhM I 5 Duffy potrebbe essere qualcosa come un'epistola prefatoria, non sono noti finora manoscritti. ${ }^{24}$

\section{Appendice III}

\section{Sulla praefatio alla traduzione latina di Ermolao Barbaro della Parafrasi degli Analitici posteriori di Temistio}

La traduzione latina della Parafrasi di Temistio da parte di Ermolao Barbaro (iuniore) (* Venezia 1453 [o 1454]; † Roma 1493) $)^{25}$ fu stampata in folio insieme alle versioni delle parafrasi temistiane di altre sei opere di Aristotele (Physica, De anima, De memoria et reminiscentia, De somno et vigilia, De insomniis, De divinatione per somnum) a Treviso il 15 febbraio del 1481 da Bartholomaeus Confalonerius e Morellus Gerardinus. ${ }^{26}$ Il prologus Themistii si legge alla carta

24 "Psellos' synopsis, however, still waits to be recovered from the surviving manuscripts": Ierodiakonou 2002, 159 .

25 Sulla biografia di Ermolao Barbaro vd. almeno Bigi 1964. Ben prima di Ermolao Barbaro, aveva tradotto in latino la parafrasi di Temistio in epoca tardoantica Vettio Agorio Pretestato (320 ca.-384), teste Boethio (Meiser 1880, 3,7-4,3): Vetius Praetextatus priores postremosque analyticos non vertendo Aristotelem Latino sermoni tradidit, sed transferendo Themistium, quod qui utrosque legit facile intellegit, ma vd. anche Hadot 1971, 41; una seconda traduzione data al XII secolo ed è di Gerardo da Cremona. Nel Xvi secolo, la versione del Barbaro servì da modello a Paolo Orsatto per la propria traduzione. Per i dati sulle traduzioni di Gerardo da Cremona e Paolo Orsatto, cfr. Todd 2003, 73-77.

26 Cfr. la relativa scheda disponibile in linea sul sito dell'Incunabula Short Title Catalogue della British Library di Londra allindirizzo http://istc.bl.uk/search/search.html?operation=record\&rsid=636133\&q=0, nonché la nota tipografica alla fine della traduzione della parafrasi della Fisica (carta kiiiir, non numerata): Themistii liber ultimus physices explicit. 
1r, preceduto dal titolo Themistii Euphradae Peripatetici Nobiliss(imi) Paraphral sis in Posteriora Analitica Aristotelis Interprete / Hermolao Barbaro Patricio Veneto, Viro Clarissimo, e suona come segue:

Facturum me superfluo iuxta perambitioseque sentio, si aristotelicarum commentationum post tales tantosque auctores interpretationem iustam aggrediar, praesertim quod haud temere multa inuenias quae illi perinde non dixerint alioquin ut omissa pauca sarcias. Tantum laboris a capite retexere simile feceris ac si Phidiae Mineruam reformare de integro uniuersam instituas ut aut amentum soleis aut ansulam crepidis aut ligulam baseis aut quid aliud pauxillum quod subsultet reponas. Quod si praetermissa morositate disputationis summas rerum per consectaria collegero iisque limatam et compendiariam enarrationem adiunxero breuitatemque et seriem philosophi ex summis uiribus persecutus imitatusque fuero uidebor utique rem nec uulgatam neque admodum inutilem noscentibus haec parasse.

Identificare con esattezza il manoscritto greco sul quale il Barbaro eseguì questa versione comporterebbe senz'altro un avanzamento delle nostre conoscenze rispetto a quanto già scritto nel 1900 da Max Wallies nella Praefatio all'edizione del testo greco di Themistii Analyticorum Posteriorum Paraphrasis per la serie berlinese dei Commentaria in Aristotelem Graeca, ma evidentemente non è questa la sede per una ricerca siffatta. Per il breve brano riportato sopra, posso confermare il dato del Wallies, secondo cui l'esemplare greco a disposizione del Barbaro sarebbe stato uno dei dodici codici dell'altera classis da lui stesso individuata nel corso della recensio della tradizione manoscritta. ${ }^{27}$ A tal proposito, infatti, nella frase d'esordio della versione del Barbaro, l'ind.

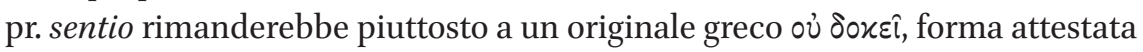
in manoscritti della seconda classe, per es. i testimoni A (= Ambrosianus G 14

/ Bartholomceus Confalonerius Brixiensis Taruisii Impressit. Un esemplare di questo incunabolo (ISTC No.: itoo12900o) è disponibile in formato digitale sul sito della Bayerische Staatsbibliothek di Monaco di Baviera all'indirizzo http://daten.digitale-sammlungen. $\mathrm{de} / \sim \mathrm{db} /$ ooo6/bsbooo6968o/images/. Si tratta dell'esemplare appartenuto al giurista, umanista e antiquario tedesco Konrad Peutinger ( ${ }^{*}$ Augusta 1465; $\dagger$ ivi 1547) come informa l'ex libris apposto sul recto della seconda carta iniziale, non numerata dell'incunabolo: Liber Conradi Peutinger Augustani / utriusque iuris doctoris $2 c$ (forse da sciogliersi in secundi, -ae intendendo così la possibile collocazione del libro nella biblioteca di Peutinger, come anche mi fa notare Luigi Orlandi). Sulla controguardia posteriore si legge l'annotazione seguente: inuidia pestiferum uirus monasticeque uite / magnum dedecus experientia docuit actiue nusquam sed passiue. 


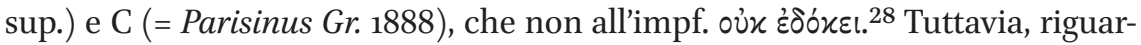
do all'identificazione delle fonti, Wallies era ben consapevole della difficoltà di ottenere risultati più certi, essendo quella del Barbaro, come quest'ultimo riconosceva nella dedica della versione al Papa Sisto IV, ${ }^{29}$ non una traduzione letterale, bensì libera e adattata ai modi della lingua d'arrivo. In his libris vertendis non modo non expressimus verbum e verbo quod interpretes indisertos Cicero meminit, sed libere et translationibus et figuris et tropis usi sumus ad morem Romanum sensibus stantibus. Lusimus arbitratu nostro, sed sententiam integram dedimus auctori. [...] In plenum non tam latinum reddere Themistium quam certare cum eo volui, scrive il Barbaro. ${ }^{30}$

Quanto e come poi egli abbia realizzato il proposito dichiarato in questa lettera dedicatoria, quello cioè di tradurre Temistio 'secondo l'uso Romano' (ad morem Romanum) e di 'gareggiare' (certare) con il retore greco, basta a dimostrarlo già la prima frase della versione: Facturum me superfluo iuxta perambitioseque sentio, nella quale è dato cogliere un'eco liviana: Facturusne operae pretium sim, si a primordio urbis res populi Romani perscripserim, nec satis scio nec, si sciam, dicere ausim... ${ }^{31}$

Né il gioco di sottili richiami alla latinitas da parte del Barbaro si esaurisce con la citazione da Livio. Anche nella frase: ut aut amentum soleis aut ansulam crepidis aut ligulam baseis aut quid aliud pauxillum quod subsultet reponas,

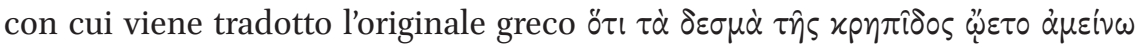
$\pi \circ \eta^{\sigma} \sigma \varepsilon เ \nu$, non può essere escluso, infatti, per la iunctura 'ansulam crepidis' una citazione da Valerio Massimo (8,12 ext. 3: de crepida et ansulis), l'altro autore latino, in qualche modo testimone del detto sutor ne ultra crepidam, oltre a

28 Cfr. Wallies 19oo, 1,3 e apparato ad loc.

29 Sotto il titolo Hermolai Barbari Patricii Veneti Zachariae Equitis Filii in Paraphrasin Themistii Peripatetici Praefatio ad Sixtum IV Pontificem Maximum, questo testo si legge nel verso e nel recto rispettivamente della seconda e terza carta iniziale, non numerate, dell'incunabolo.

30 Cfr. Wallies 1900, XIV.

31 Liv., Praef. 1. L'allusione a Livio da parte del Barbaro non si limita tuttavia alla sola ripresa della forma participiale (facturum) posta in esordio. Anche la frase uidebor utique rem nec uulgatam neque admodum inutilem noscentibus haec parasse sembra, infatti, riecheggiare le parole dello storico latino poco più avanti nella stessa Praefatio $\left(\S_{2}\right)$ : quippe qui cum veterem tum vulgatam esse rem videam. Inoltre, a me pare che nel tradurre in latino il pensiero di Temistio, il quale temeva che il suo progetto di esegesi agli Analitici posteriori di Aristotele riuscisse inutilmente ambizioso, il Barbaro abbia voluto anche imitare più in generale lo stato d'animo di Livio rispetto all'antichità della materia trattata e ai novi scriptores, i quali cercano o di accertare meglio la verità dei fatti narrati oppure di superare nell'arte dello scrivere gli storici del passato, cfr. Praef. 1-2. 
Plinio il Vecchio, oggetto com'è noto di cure filologiche da parte dello stesso Barbaro. $^{32}$

La Praefatio alla traduzione della parafrasi temistiana degli Analitici secondi che Ermolao Barbaro dedica da Venezia nel 1480 al pontefice Sisto IV (14711484), "un francescano letterato che aveva insegnato in diverse università dell'Italia del nord e che viveva circondato da teologi e artisti",33 offre inoltre informazioni su come un esponente dell'Umanesimo greco-latino nell'Italia della fine del Xv secolo definiva i concetti di parafrasi, metafrasi ed ecfrasi. Dopo aver fornito alcuni dettagli sulla figura di Temistio, scrive, infatti, il Barbaro quasi alla fine della prefazione:

Est autem paraphrasis exercitamenti apud rhetoras genus. Ea finitur a græcis hoc modo, ut sit quæ narrationi proportione respondeat: aut sic in qua uertimus aliorum scripta. Non in alia quæ humiliora grandioraue sint: ut a metaphrasi differat: sed in paria sensu modo seruato [...]. ${ }^{34}$ Neque ego paraphrasin esse interpretationem tantum uolo sed circa eosdem sensus certamen atque æmulationem. Dictus est \& a quibusdam ecphrastes. Est autem ecphrasis mera puraque enarratio.

Sulla scia della tradizione retorica antica, il Barbaro intende per parafrasi una traduzione rispondente 'in proporzione al dettato' dello scritto altrui. È sostanzialmente una questione di quantità di parole: quante nell'originale, tante nella parafrasi; metafrasi corrisponderebbe a una riscrittura, più o meno libera, visto che in essa gli aliorum scripta diventano, a seconda dei casi, 'più umili o più grandi'; ecfrasi, infine, nel significato bizantino di descrizione pura e semplice, per es. di un oggetto o di un monumento.

\section{Ringraziamenti}

I dati contenuti nell'articolo sono stati raccolti nell'ambito del lavoro al progetto Co6 'Aristoteles-Manuskripte in Unterricht und Auslegungspraxis', guidato dal Professor Christian Brockmann e parte del SFB 950 'Manuskriptkulturen in Asien, Afrika und Europa' /CSMC dell'Università di Amburgo, finanziato dalla Deutsche Forschungsgemeinschaft (DFG). Alcuni punti del lavoro si

32 Nelle Castigationes Plinianae stampate a Roma nel 1492.

33 Così Fellay 2017, 121.

34 Nelle righe omesse segue la citazione di due passi dell'Institutio oratoria di Quintiliano $(1.9 .2-3$ e 10.5.5). 
sono giovati di proficue discussioni con Nikos Agiotis, Frederick Lauritzen, Tommaso Migliorini, Luigi Orlandi, Tommaso Raiola e Amneris Roselli che ringrazio.

\section{Bibliografia}

Bianconi, D. (2003). Eracle e Iolao. Aspetti della collaborazione tra copisti nell'età dei Paleologi. Byzantinische Zeitschrift 96, pp. 521-558.

Bianchi, N. e Schiano, C., edd. (2016). Fozio. Biblioteca. Introduzione di L. Canfora, nota sulla traduzione manoscritta di S. Micunco, Pisa.

Bigi, E. (1964). “Ermolao Barbaro", in: Dizionario biografico degli italiani. Vol. 6, Roma.

Cacouros, M., ed. (1992). Le Commentaire de Théodore Prodrome au second livre des Analytiques Postérieurs d'Aristote. Texte (édition princeps et tradition manuscrite) et étude logique du commentaire de Prodrome. Thèse de doctorat, Paris IV.

Cacouros, M. (1994). Un commentaire byzantin inédit au deuxième livre des Seconds Analytiques attribuable à Jean Chortasménos. Revue d'Histoire des Textes 24, pp. 150-198.

Capocci, V. (1958). Codices Barberiniani Graeci. Tomus I. Codices 1-163. Città del Vaticano. Chantry, M., ed. (2001). Scholia in Aristophanem. Pars III. Scholia in Thesmophoriazusas, Ranas, Ecclesiazusas et Plutum, fasc. $I^{b}$ continens scholia recentiora in Aristophanis Ranas. Groningen.

De Falco, V., ed. (1926). Ioannis Pediasimi in Aristotelis Analytica scholia selecta. Napoli.

De Falco, V. (1928). Altri scolii di Giovanni Pediasimo agli Analitici. Byzantinische Zeitschrift 28, pp. 251-269.

Duffy, J. M., ed. (1992). Michaelis Pselli Philosophica Minora. Vol. I: Opuscula logica, physica, allegorica, alia. Stuttgart-Leipzig.

Ebbesen, S. (2012). Review on F. A. J. de Haas, M. Leunissen and M. Martijn (eds.), Interpreting Aristotle's Posterior Analytics in Late Antiquity and Beyond, BostonLeiden 2010. Aestimatio 9, pp. 355-366.

Fellay, J. B. (2017). Raffaello, l'antitesi di Lutero. La Civiltà Cattolica 3998, anno 168, 14/28 gennaio, pp. 120-132.

Golitsis, P. (2008). Georges Pachymère comme didascale. Essai pour une reconstruction de sa carrière et de son enseignement philosophique. Jahrbuch der österreichischen Byzantinistik 56, pp. 53-68.

Hadot, P. (1971). Marius Victorinus: Recherches sur sa vie et ses oeuvres. Paris.

Ierodiakonou, K. (2002). Psellos' Paraphrasis on Aristotle's De interpretatione. In: K. Ierodianakou (ed.), Byzantine Philosophy and its Ancient Sources, Oxford, pp. $157-181$. 
Meiser, C., ed. (1880). Anicii Manlii Severini Boetii commentarii in librum Aristotelis $\pi \varepsilon p i$

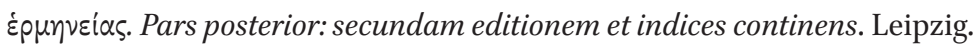

Moraux, P. (1979). Le commentaire d'Alexandre d'Aphrodise aux «Seconds Analytiques» d'Aristote. Berlin-New York.

Todd, R. B. (2003). Themistius. In:V. Brown, J. Hankins and R. A. Kaster (eds.), Catalogus translationum et commentariorum: Mediaeval and Renaissance Latin Translations and Commentaries. Annotated Lists and Guides, Washington, DC, pp. 57-102.

Tosi, R. (1991). Dizionario delle sentenze latine e greche. Milano. 